\title{
DIFERENCIAS ENTRE LA EDUCACIÓN UNIVERSITARIA PÚBLICA Y PRIVADA COSTARRICENSE: RENDIMIENTOS Y CALIFICACIÓN DEL TRABAJO
}

\author{
DIFFERENCES AMONG COSTA RICAN PUBLIC AND PRIVATE TERTIARY \\ EDUCATION: RETURNS AND OVEREDUCATION
}

\author{
Laura C. Blanco ${ }^{1}$ \\ María José Sauma Chacón²
}

Recibido: 15/09/2019

Aprobado: 08/03/2020

RESUMEN

Se estimaron los niveles de sobreeducación para la población costarricense graduada de estudios superiores utilizando la Encuesta Nacional de Hogares 2011-2017. Posteriormente, se estimaron ecuaciones de Mincer mediante mínimos cuadrados ordinarios para analizar la relación existente entre el salario y el tipo de centro educativo, el área de estudio y el tipo de emparejamiento laboral. Se encuentra que estudiar en una universidad pública se asocia con un premio que oscila entre 4,7\% y 9,5\%. Este premio, además, varía por área de estudio, aunque se encuentra que las universidades públicas mantienen su ventaja en la mayoría de los casos. Para las cohortes de mayor edad, el premio por estudiar en una universidad pública desaparece. Además, se encuentra que no existe un problema por genuina sobreeducación en el mercado laboral costarricense, aunque sí existe un porcentaje importante de personas aparentemente sobreeducadas, el cual es mayor entre quienes estudiaron en universidades públicas.

PALABRAS CLAVE: RESULTADOS EDUCATIVOS, EDUCACIÓN, EDUCACIÓN SUPERIOR, CAPITAL HUMANO, FUNCIONES DE INGRESO DE MINCER, TASAS DE RETORNO DE LA EDUCACIÓN, DECISIÓN OCUPACIONAL.

CLASIFICACIÓN JEL: I21, I23, I26, J24

\section{ABSTRACT}

Overeducation rates were estimated for the Costa Rican graduate working population using the National Household Survey 2011-2017. Further on, Mincerian wage equations were estimated using ordinary least squares to analyze the existing relationship between wages 
and the type of university attended, degree subject, area and skill mismatches. Results show that attending a public university is associated with a wage premium ranging from $4.7 \%$ to $9.5 \%$. This premium varies by degree subject, but public universities exhibit an advantage in most cases. For elderly cohorts, the premium associated with being a graduate from a public university disappears. Genuine overeducation is not found to be a problem in the Costa Rican labor market, despite the existence of a considerable percentage of apparently overeducated graduates; and this percentage is found to be higher among public universities alumni.

KEYWORDS: EDUCATIONAL OUTCOMES, SCHOOLING, HIGHER EDUCATION, HUMAN CAPITAL, MINCER EARNINGS FUNCTION, RETURNS TO EDUCATION, OCCUPATIONAL CHOICE

JEL CLASSIFICATION: I21, I23, I26, J24.

\section{INTRODUCCIÓN}

La teoría del capital humano (Becker, 1962 y 1993, Mincer, 1974; Schultz, 1961) provee el marco teórico fundamental para comprender la determinación de los salarios en el mercado laboral. Para el caso costarricense, la educación es el factor más determinante para explicar la desigualdad en el ingreso salarial, ya que sus rendimientos son crecientes (Fernández \& Del Valle, 2011; Gindling \& Trejos, 2005). No obstante, solo el 27,6\% de la población entre los 18 y los 24 años asiste a centros educativos universitarios (Programa Estado de la Nación en Desarrollo Humano Sostenible [PEN], 2019), lo que acrecienta aún más la desigualdad, pues las personas con estudios superiores representan al grupo más pequeño y con mejores resultados salariales.

Lo anterior suscita discusiones en foros políticos y sociales sobre el uso de recursos públicos destinados a la educación superior, así como los esfuerzos privados que las personas realizan para educarse $^{3}$ (Arguedas Ortiz, 2015; Madrigal, 2015), pues si bien las universidades públicas antes eran percibidas como mecanismos de movilidad social, cada vez más son vilipendiadas como élites. El creciente discurso popular cuestiona el papel de las universidades públicas en contraposición con las privadas, siendo el supuesto implícito que ambos tipos de instituciones son sustitutas perfectas. Este supuesto, sin embargo, merece ser revisado, pues la estructuración de la oferta de estudios superiores provista por instituciones públicas y privadas difiere entre sí (PEN, 2019) y, por tanto, también podrían diferir los resultados económicos que dichas instituciones ofrecen a sus personas graduadas (PEN, 2019). Así, resulta de interés, e inclusive necesario ante el contexto nacional, estudiar si efectivamente existen diferencias en la calidad de la educación terciaria en el país. Medir los resultados económicos de las personas graduadas de las universidades públicas y privadas es una primera aproximación para entender cuán profundamente pueden diferir los servicios que unas y otras ofrecen.

El objetivo de este artículo consiste en determinar si existen diferencias en las tasas de sobreeducación y en los rendimientos de la educación de las personas graduadas de universidades públicas frente a las privadas. Para ello, se estiman los niveles de calificación de las personas ocupadas y graduadas de estudios superiores, utilizando la Encuesta Nacional de Hogares(ENAHO) para el período 2011-2017. Posteriormente, se estiman ecuaciones de ingreso con el fin de obtener los rendimientos brutos asociados a las áreas de estudio según el tipo de institución a la que asistieron. Se encuentra que, efectivamente, existe un premio promedio de alrededor de 6\% por graduarse de una institución pública y este persiste por parte importante de la vida laboral, aunque

3 El financiamiento del Estado a las universidades públicas para 2018 ascendió a más de $\$ 496$ mil millones (CONARE, 2019), mientras que el presupuesto a CONAPE, quien financia los créditos para estudios superiores, fue de $\$ 33,6$ mil millones para ese mismo año (CONAPE, 2017). 
desaparece completamente para la cohorte con más de 50 años. El premio, además, varía en magnitud según el área de estudio, pero se mantiene para la mayoría de estas e, incluso, se observan tasas de castigo por estudiar humanidades, servicios o medicina en instituciones privadas. Lo anterior podría resultar relevante al discutir políticas públicas en torno, por ejemplo, a la definición de criterios de excelencia, el financiamiento o la coordinación para aumentar las productividades en el mercado laboral a través de la educación superior.

El documento se estructura de la siguiente manera: en la sección II se presentan los antecedentes contextuales y teóricos que motivan la discusión. En la sección III se describe la base de datos a partir de la cual se construye la muestra, así como la metodología a utilizar, mientras que la sección IV presenta los resultados y la sección V concluye.

\section{ANTECEDENTES}

\section{Educación universitaria en Costa Rica}

En Costa Rica existen solo 5 instituciones de educación superior públicas, en comparación con 53 privadas y 6 internacionales (PEN, 2019). Sin embargo, las primeras reciben alrededor del 50\% de la matrícula universitaria, lo que significa que comparten similarmente el peso de la demanda educativa con las instituciones privadas (PEN, 2019). Si se utiliza la nomenclatura de Geiger (1988), Costa Rica aparenta poseer un sistema de educación superior de sectores público y privado paralelos, el cual se define como aquel en que las universidades, aunque diferentes entre sí, tienen el mismo peso a nivel nacional y ofrecen educación de valor equivalente. Empero, existe evidencia que señala que la educación universitaria privada puede no ser sustituta perfecta de la pública.

Las universidades públicas en Costa Rica son reguladas por el Consejo Nacional de Rectores (CONARE), mientras que las privadas lo son por el Consejo Nacional de Enseñanza Superior Universitaria Privada (CONESUP) y ambas instituciones están conformadas por diferentes miembros (CONARE, 2018; MEP, 2001). CONARE se encarga de la planificación y desarrollo de las instituciones públicas de educación superior. CONESUP, en cambio, se limita a aprobar las propuestas curriculares que cumplan con los criterios mínimos de diseño curricular, vigilar e inspeccionar a las contrapartes privadas, con el fin de "garantizar que los estudiantes que se matriculen en las universidades privadas costarricenses reciban una educación universitaria de excelencia, que sea congruente con la propuesta curricular aprobada por el CONESUP" (MEP, 2001, art.2a). La función del CONESUP como órgano rector es muy limitada y ha sido interpretada por el Ministerio de Educación Pública (2018) como el mandato de asegurar que cada universidad "ofrezca una educación de valor equivalente". Sin embargo, es imposible ignorar el hecho de que las instituciones públicas y privadas están reguladas por diferentes consejos con diferentes lineamientos, metas y potestades.

Las universidades públicas giran alrededor de "la difusión del conocimiento", "el desarrollo del país", "conocimientos y bienestar", "desarrollo sostenible" y "acceso para todos" (Instituto Tecnológico de Costa Rica, 1983, art. 1; Universidad de Costa Rica, 1974, art. 1; Universidad Nacional , 2015, art.3; Universidad Estatal a Distancia, 2000, art. 2 y Universidad Técnica Nacional, 2010, art. 4), mientras que las instituciones privadas, si bien pueden compartir estos objetivos, son empresas $y$, como tales, deben responder a sus accionistas y su objetivo de maximización de beneficios (Ginsburg et al., 2012; McPherson, 1981). Los institutos privados pueden buscar el bienestar y el desarrollo, pero su rendición de cuentas es distinta (White, 2003). Además, si bien pueden existir entes reguladores de control de calidad, estos no siempre funcionan o garantizan la equivalencia de títulos, ya que puede que no exijan o vigilen debidamente a las universidades privadas, inclusive dando pie a que se falsifiquen datos y estos no sean verificados (Welch, 2007). 
Un ejemplo de esto es el reciente cierre por parte de CONESUP de la Universidad Cristiana del Sur (Vizcaíno \& Cerdas, 2018), ligada a una figura política y denunciada por aceptar estudiantes sin título de bachillerato de secundaria y contratar docentes que no cumplían con los requisitos exigidos (Cerdas \& Chinchilla, 2017).

Si bien las universidades públicas y privadas son reguladas por entes diferentes $y$, por tanto, no existen bases de datos de dichos supervisores que permitan comparar su desempeño (PEN, 2019), un instrumento aceptado para realizar este ejercicio son las mediciones internacionales. La tabla 1 muestra los primeros diez puestos en el país de la clasificación Webometrics (Cybermetrics Lab, 2019). En esta aparecen 56 de las 64 instituciones de educación superior costarricenses y, por tanto, es la lista más completa y actualizada (a junio de 2019) disponible. La Universidad de Costa Rica aparece en el primer lugar de la clasificación nacional y, con excepción de la Universidad Técnica Nacional, de más reciente creación, todas las otras universidades públicas se encuentran antes que la primera de las privadas, la Universidad Latina de Costa Rica, la cual ocupa el puesto 8. Además, a nivel de la región latinoamericana, la Universidad de Costa Rica ocupa la posición 28 y la Universidad Latina de Costa Rica, la 709. Lo anterior evidencia una marcada diferencia entre las instituciones públicas y privadas. Obviamente, estas distancias se acentúan si se compara con otras universidades hacia el final de la lista, como la Universidad Teológica de América Central Monseñor Óscar Arnulfo Romero o la Universidad Continental de las Ciencias y las Artes, quienes ocupan las posiciones 3686 y 3825 en la región latinoamericana, respectivamente. Las universidades internacionales, por el contrario, se hallan más próximas a las públicas $y$, es de suponer, que se parecen más a estas en sus objetivos y funcionamiento.

Una característica fundamental de la universidad es que esta es un centro de producción de conocimiento. Sin embargo, solo las universidades públicas parecen cumplir con esta característica esencial. En la clasificación QS Latin American University Rankings 2018 (Quacquarelli Symonds

TABLA 1

\section{CLASIFICACIÓN DE UNIVERSIDADES COSTARRICENSES}

(10 primeros puestos) Año: 2019

\begin{tabular}{|c|c|c|c|c|}
\hline Universidad & Tipo & País & América Latina & Mundo \\
\hline Universidad de Costa Rica & Pública & 1 & 28 & 844 \\
\hline Universidad Nacional & Pública & 2 & 121 & 2235 \\
\hline $\begin{array}{l}\text { Centro Agronómico Tropical de Investigación y } \\
\text { Enseñanza }\end{array}$ & Internacional & 3 & 160 & 2558 \\
\hline Instituto Tecnológico de Costa Rica & Pública & 4 & 219 & 3044 \\
\hline INCAE Business School & Internacional & 5 & 350 & 3917 \\
\hline Universidad Estatal a Distancia Costa Rica & Pública & 6 & 365 & 4070 \\
\hline University for Peace & Internacional & 7 & 437 & 4451 \\
\hline $\begin{array}{l}\text { Universidad Latina de Costa Rica (Universidad } \\
\text { Interamericana) }\end{array}$ & Privada & 8 & 709 & 7250 \\
\hline Instituto Interamericano de Derechos Humanos & Internacional & 9 & 795 & 8652 \\
\hline $\begin{array}{l}\text { Universidad Latinoamericana de Ciencia y Tecnología } \\
\text { Costa Rica }\end{array}$ & Privada & 10 & 809 & 8776 \\
\hline
\end{tabular}

Fuente: Webometrics (2019) 
Limited, 2019), que puntúa la investigación con base en Scopus (Elsevier B.V., 2019), todas las universidades privadas aparecen con un bajo índice de producción de investigación, mientras que las públicas aparecen con índices de alto (Universidad de Costa Rica) a medio (Universidad Nacional). Otra de las diferencias principales es que los centros universitarios públicos son multiárea, con la única excepción del Instituto Tecnológico de Costa Rica, especializado en ciencias, tecnología, ingeniería y matemáticas, mientras que las instituciones privadas son relativamente pequeñas con una oferta académica limitada y concentrada en pocas áreas, así como con baja matrícula y graduaciones, aunque aquí también hay algunas excepciones (PEN, 2019). Estas universidades tienden a ubicarse geográficamente cerca de alguna sede de una universidad pública con una oferta educativa similar para capturar su exceso de demanda (PEN, 2019) ${ }^{4}$. Lo anterior sugiere que, efectivamente, podrían existir diferencias fundamentales entre el tipo de formación que pueden ofrecer unos y otros centros de estudio.

Los resultados de los exámenes del International Foundations of Medicine (IFOM) que se utilizan para admitir al estudiantado de medicina en puestos interinos en los hospitales y el examen de incorporación al Colegio de Abogados son dos ejemplos de estas diferencias. En el primer caso, durante los últimos años, el 98\% de la población estudiantil proveniente de la Universidad de Costa Rica (única universidad pública que ofrece la carrera de medicina) aprobó el examen, mientras que ninguna otra universidad (todas privadas) alcanzó el 90\% de aprobación, e inclusive, algunas se encuentran por debajo de 50\% (Ávalos, 2017; Ávalos, 2018; Solís Ramírez, 2018). Además, los resultados de quienes estudian en la Universidad de Costa Rica son los únicos que se encuentran por encima del promedio internacional. Respecto al examen de incorporación al Colegio de Abogados de Costa Rica, hace dos años diecinueve universidades privadas no lograron alcanzar un 50\% de aprobación, y entre estas, diecisiete estuvieron por debajo de 34\%. En 2018, todas las universidades tuvieron una tasa de aprobación inferior al 23\%, con excepción de la Universidad Libre de Derecho y la Universidad de Costa Rica (Cerdas, 2017; Cerdas, 2019).

Ante este contexto, es natural cuestionarse la calidad de la educación en dichos centros de estudio. Este cuestionamiento es relevante, además, por los recursos públicos involucrados, ya que no solamente las universidades estatales son financiadas por el Estado costarricense, sino que también las universidades privadas reciben exenciones de impuestos y pagan una tasa reducida del impuesto al valor agregado del 2\%, en lugar del 13\% que le corresponde, en promedio, a los demás servicios. Argumentos respecto a la tasación de la educación (Flores Curiel, 2016; Levy, 1982) asociaciones público-privadas en el área (Bruce, 1983; Hodge \& Greve, 2007; Patrinos, 2009) y la importancia de la pluralidad en la oferta educativa (Levin, 1991; Stiglitz, 1974) se han desarrollado a lo largo del mundo y si bien no son el enfoque aquí, constituyen un tema de mucha relevancia para la política pública (Doyle, 1980).

Por último, las personas invierten en educación esperando que esta les reditúe mayores rendimientos a lo largo de su vida laboral, al permitirles optar por trabajos de mayor calificación. En Costa Rica, los rendimientos asociados con la educación son crecientes (Fernández \& Del Valle, 2011) y pueden oscilar entre 10\% y hasta un 30\% para el grado universitario, dependiendo del método de estimación (Mata \& Valverde, 2006). En los países desarrollados, donde un mayor porcentaje de la población asiste a la educación superior, la discusión sobre su financiamiento muchas veces gira en torno a la sobreeducación (sobrecalificación), i.e., el exceso de inversión en educación, medido a través de los años de escolaridad (nivel de competencia o nivel educativo), que provoca que personas con estudios universitarios deban ubicarse en puestos que no requieren dichos estudios debido al exceso de oferta de personas graduadas de la universidad (Alpin, Shackleton \& Walsh, 1998; Boll, Leppin, Rossen, \& Wolf, 2016; Chevalier, 2003; Chevalier \& Lindley, 2009; Quintini, 2011). Esta

4 Si bien puede haber excepciones, este es el patrón identificado por PEN (2019). 
sobreeducación puede estar reflejando diferencias en las habilidades: las personas menos hábiles se ubicarían en puestos con requerimientos inferiores a los correspondientes con su grado académico, pero acordes con su nivel de habilidades, de forma que su sobreeducación sería aparente. En contraste, las personas genuinamente sobreeducadas reportarían insatisfacción en su trabajo, al no hacer un uso apropiado de sus habilidades (Chevalier, 2003). Quienes reportan sobreeducación reciben un salario inferior al de personas con su mismo nivel de educación y en un puesto acorde con dicha educación (Chevalier, 2003; Chevalier \& Lindley, 2009; Dolton \& Silles, 2001; Verdugo \& Verdugo, 1988; Verhaest \& Omey, 2006).

Lo anterior plantea un segundo aspecto a analizar para el caso costarricense, pues no solo puede encontrarse diferencias respecto a los rendimientos asociados al salario, sino que también podría haber diversas tasas de sobreeducación en una y otra institución.

\section{Diferencias en la calidad educativa}

El término calidad de la educación se refiere al conocimiento y las habilidades analíticas que se obtienen mediante el proceso educativo (Hanushek, 2002). Estos conocimientos y habilidades forman parte del capital humano, el cual es un factor de producción (Becker, 1962). La calidad de la educación se encuentra positivamente relacionada con el crecimiento y desarrollo económico de un país y estas tasas de crecimiento se acentúan cuando esta educación es mayoritariamente pública (Hanushek, Ruhose \& Woesmann, 2015). Además, si se parte de una misma cantidad de educación, la diferencia en los rendimientos de la educación debe ser a causa de su calidad: mayor capital de conocimiento significa mayores retornos (Hanushek, Ruhose \& Woessmann, 2015). Mejores resultados en la educación pública de los países se relacionan con una menor desigualdad económica y una mayor movilidad laboral (Davies, Zhang \& Zeng, 2005). Asimismo, entre mejor sea la educación pública, mayor es la participación en el mercado laboral global (Marginson, 2007) y, conforme mejora el sistema educativo público, aumenta la cantidad de personas que concluyen la educación formal (Fioroni, 2010). Dado que en Costa Rica se cuenta con un sistema de sector público y privado paralelos en la educación superior, cabe analizar si los diferentes sectores difieren en su impacto con respecto a las posibilidades de ingreso $y$, eventualmente, de crecimiento económico.

En Costa Rica, los estudios realizados en torno a la calidad de la educación se han enfocado en secundaria ${ }^{5}$. Se ha encontrado que existe una brecha considerable entre las tasas de aprobación de secundaria de los colegios privados respecto a los públicos (PEN, 2015), señalizando que, aunque se aplique el mismo programa de estudios de secundaria, los resultados entre los tipos de institución no son equivalentes. Asimismo, existe una diferencia significativa entre los resultados del estudiantado de colegios públicos y privados en las pruebas PISA, siendo estos últimos los que se desempeñan mejor (Fernández \& Del Valle, 2013). Sin embargo, dichos resultados se ven altamente afectados por las condiciones socioeconómicas de cada estudiante, por lo cual los logros académicos no dependen únicamente del tipo de institución educativa (Montero, Rojas, Zamora \& Rodino, 2012). Un ejemplo similar es el de las tasas de deserción escolar, las cuales son casi nulas para los institutos privados y relativamente altas para los públicos (PEN, 2015). Así, la calidad educativa parece ser uno de los factores que afecta la deserción escolar, pero los resultados no son tan concluyentes cuando esta se cruza con la condición socioeconómica del estudiantado (Hanushek, Lavy \& Hitomi, 2006). Cabría preguntarse si el desempeño observado entre las instituciones públicas y privadas a nivel de secundaria se invierte para el caso de los estudios superiores, dado que las universidades privadas aparecen peor calificadas en las clasificaciones internacionales que las universidades públicas y estas últimas tradicionalmente gozan de mayor reputación que las privadas.

5 Existen estudios sobre el desempeño educativo en universidades, pero estos no permiten comparar el desempeño estudiantil por tipo de universidad. Tanto Montero, Villalobos \& Valverde (2007) como Abarca, Leiva, Robalino, \& Saborío (2016) analizan los factores que inciden sobre el desempeño estudiantil utilizando muestras de la Universidad de Costa Rica. 
Fuera del territorio costarricense, el estudio de las diferencias en la calidad educativa también se ha centrado en la educación secundaria. La educación secundaria privada generalmente ofrece días escolares más largos, una razón estudiante/docente más baja y un segundo o tercer idioma, por lo que es esperable el desarrollo de conocimientos y habilidades sea mayor (Flores Curiel, 2016). En Canadá, por ejemplo, las escuelas privadas son más efectivas y eficientes que las escuelas públicas (Paquette, 2005). En Estados Unidos, se ha encontrado evidencia de que las personas que asistieron a educación privada y a centros educativos localizados en los suburbios (que, si bien no son necesariamente privados, gozan de mayor financiamiento al ubicarse en zonas de mayor privilegio) reportan mayores salarios que las que no (Sandy \& Duncan, 1996; Sexton \& Nickel, 1992). Sin embargo, este resultado se atribuye también a las características familiares, aunque no es de negar que las personas invierten en educación privada porque esperan obtener beneficios futuros de ella.

En torno a la calidad de la educación terciaria, si bien los estudios son escasos, existen otros indicadores de estas diferencias. Por ejemplo, en el Reino Unido, las políticas de financiamiento a la educación superior son utilizadas como un mecanismo para que las universidades, tanto públicas como privadas, compitan por recursos con base en su calidad. Se utiliza el Marco de Excelencia de la Investigación para realizar mediciones de calidad e impacto de la investigación que producen las universidades y estas compiten por fondos con base en su desempeño en dichas evaluaciones (Research Excellence Framework, 2019). Similarmente, la Agencia de Estadística de Educación Superior (HESA, 2019) recopila toda la información posible de las universidades y el desempeño de sus estudiantes, con el fin de publicar información relevante sobre la calidad educativa y así optimizar la distribución de recursos entre los centros de estudio. Estos tipos de estándares de medición de la calidad educativa son inexistentes en Costa Rica. Si bien CONARE realiza encuestas a las personas recién graduadas de las universidades costarricenses, esta es una base de datos que únicamente se utiliza para recopilar información, pero no para realizar políticas públicas ni para generar incentivos para que las instituciones eleven su calidad educativa.

En Estados Unidos, se da el caso que las universidades privadas intentan competir como instituciones selectivas, utilizando el costo de matrícula, para llamar la atención de las y los estudiantes más brillantes (Anderson, 1975). En conjunto, al hacer esto y finalmente obtener al mejor estudiantado, empiezan a mejorar su reputación y con ello logran atraer la atención y la preferencia de las otras personas y posibles estudiantes. En Costa Rica, si bien el costo de las universidades privadas es mayor que el costo de las universidades públicas, la competencia por "institución selectiva" se puede dar por algo más que por el costo de matrícula: el examen de admisión. Para poder estudiar en una universidad estatal, se requiere haber obtenido una nota mínima de un examen de admisión que evalúa habilidades cuantitativas y lingüísticas. Para las universidades privadas, en cambio, basta con tener el título en educación secundaria y el financiamiento.

Esta selectividad puede generar un efecto de pares que amplifique las diferencias entre universidades. Al producir efectos positivos sobre sus pares, las personas más hábiles logran incrementar, no solo sus logros académicos, sino también los de sus pares en la escuela a la que asisten, de forma que la calidad de una escuela está determinada por la media de la habilidad entre el cuerpo estudiantil; esto también puede incidir sobre el financiamiento de los centros educativos si estos ganan mayor renombre (Hoyt \& Lee, 1998). Dicho efecto podría observarse en Costa Rica, en tanto las universidades privadas capturan la matrícula residual de las universidades públicas e instalan sus sedes en cantones donde ya se cuenta con la presencia de una universidad pública (PEN, 2019). Es decir, si el filtro para seleccionar al estudiantado en la universidad pública es el examen de admisión y las privadas capturan su demanda residual, podría suponerse que quienes ingresan a las universidades públicas tienen mayores habilidades y esto produciría un efecto de pares sobre su población.

También se ha encontrado que existe una relación positiva entre los programas que requieren capacidades más altas y la educación superior pública: los países que más ofrecen programas basados en la teoría y diseñados para proporcionar calificaciones suficientes para ingresar a programas 
de investigación avanzada y profesiones con altos requisitos de habilidades tienen ingresos per cápita más altos y un menor porcentaje de estudiantes matriculados en la educación superior privada independiente (Reisz \& Stock, 2012). Como se mencionó, la brecha de investigación entre las universidades públicas y privadas costarricenses sugeriría que quienes se han preparado en las primeras tendrían una mayor probabilidad de ingreso a estos programas de investigación avanzada, señalando nuevamente la existencia de diferencias fundamentales entre ambos sistemas educativos.

De existir estas diferencias en la calidad educativa de unas y otras universidades, es de suponer que se verían reflejadas en diversos indicadores económicos, una vez que la persona se gradúe y se inserte al mercado laboral, pues quienes se gradúen de las universidades de mejor calidad deberían insertarse con mayor éxito al mercado laboral. Esta es la hipótesis que se somete a prueba en esta investigación, tal y como se explica en la sección metodológica.

\section{DATOS Y MÉTODOS}

A continuación, se presenta una descripción de la muestra utilizada, con el fin de contextualizar el problema. Posteriormente, se describen las metodologías empleadas para cuantificar los tipos de emparejamientos laborales en el mercado costarricense, así como las estimaciones de las ecuaciones salariales realizadas.

\section{Datos}

Se utilizaron datos de la ENAHO para el período 2011-2017. Esta es una muestra de representación nacional que recolecta información sobre los hogares y sus miembros. Para el período, se cuenta con información de 110392 personas ocupadas. De estas, 17780 observaciones corresponden a personas con algún grado universitario. Después de depurar la base de datos y eliminar las observaciones con información incompleta o inconsistente, se obtuvo una muestra balanceada de 14489 observaciones, de las cuales 5722 pertenecen a personas graduadas de centros educativos superiores públicos y las otras 8767 , de centros educativos superiores privados. Es decir, 39,5\% de la muestra se graduó de universidades públicas. Este dato es consistente con las estadísticas de graduaciones ${ }^{6}$. Por ejemplo, en 2018, se otorgaron 44871 diplomas universitarios, de los cuales 37,8\% correspondían a universidades públicas (PEN, 2019). Ahora bien, es de esperar que el porcentaje de la muestra (i.e., 39,5\%) sea levemente mayor al de las graduaciones más recientes, porque este es mayor para las cohortes de más edad. Debe recordarse, además, que la base de datos es representativa de la población a nivel nacional. De este total de observaciones, 12420 son personas con grado universitario, es decir, bachillerato o licenciatura, y el resto pertenecen a la población con posgrado.

La tabla 3 presenta las estadísticas descriptivas para la muestra balanceada utilizando los respectivos ponderadores poblacionales. La mayoría de la población graduada se quedó con un grado universitario $(85,1 \%)$, tal que apenas una minoría $(14,9 \%)$ cuenta con posgrado (columna 1 ).

6 Debe aclararse, además, que las tasas de graduación difieren de las tasas de matrícula. Si bien las universidades públicas representan cerca de la mitad de la matrícula, estas representan menos del 40\% de las graduaciones (PEN, 2019). 
TABLA 2

TAMAÑO DE LA MUESTRA, SEGÚN ÚLTIMO TÍTULO OBTENIDO Y TIPO DE CENTRO EDUCATIVO. Período: 2011-2017

\begin{tabular}{lrrr}
\hline \multirow{2}{*}{ Último título obtenido } & \multicolumn{3}{c}{ Tipo de centro educativo } \\
\cline { 2 - 4 } & \multicolumn{1}{c}{ Total } & Público & Privado \\
\hline Total & 14489 & 5722 & 8767 \\
Grado & 12420 & 4914 & 7506 \\
Posgrado & 2069 & 808 & 1261 \\
\hline
\end{tabular}

Fuente: Elaboración propia con base en los datos de la ENAHO

Esta población es relativamente joven, por lo que le resta alrededor de la mitad de su vida laboral. Sin embargo, debe notarse que la población graduada de universidades públicas (columna 2) es significativamente mayor en edad (40,32 años) que la graduada de universidades privadas (37,45 años) (columna 3) y esto se refleja en una mayor experiencia potencial para el primer grupo. Esta diferencia se debe a la proliferación de universidades privadas entre 1983 y 2000 y a la mayor tasa de graduación de estas en los últimos años (PEN, 2019), lo que reduce el peso de las universidades públicas entre las generaciones más jóvenes. La cohorte con mayor tamaño corresponde al grupo con edades entre 30 y 39 años $(32,4 \%)$, seguido del grupo con edades inferiores a los 30 años $(25,2 \%)$ (columna 1). En dichas cohortes, las personas graduadas de universidades privadas representan $64 \%$. En contraste, quienes se graduaron de estas universidades en la cohorte con 50 años o más representan apenas el 45\%. Quienes se hallan en sus cuarenta representan menos de un cuarto de la población (22,9\%) y el grupo de mayores de 50, al 19,5\% (columna 1), lo que es esperable, ya que a partir de esa edad las personas se empiezan a pensionar.

La gran mayoría de las personas se encuentran satisfechas con su trabajo $(97,4 \%)$ y no desean cambiarlo (95,6\%) (columna 1), aunque el nivel de insatisfacción es levemente mayor entre quienes se graduaron de universidades privadas (columna 3) y esta diferencia es estadísticamente significativa. Una situación similar se observa para quienes desean cambiar de trabajo. En ambos grupos, la mayoría reporta estabilidad laboral, gozar de seguro y trabajar en el sector público, aunque la población egresada de centros privados indica tener una mayor probabilidad de contar con trabajo permanente. En forma similar, la mayoría trabaja para empresas medianas o grandes (columna 1), aunque esto es levemente más probable para quienes se graduaron de universidades públicas (columna 2), mientras que quienes se graduaron de privadas (columna 3) tienen una mayor probabilidad de trabajar en microempresas.

Las poblaciones difieren un poco más respecto a sus características sociodemográficas, aunque estas diferencias no son tan amplias. En general, la mayoría de profesionales son mujeres (54,5\%), costarricenses $(93,8 \%)$, tienen pareja $(51,4 \%)$, no hablan un segundo idioma $(63,8 \%)$, laboran en la región Central $(77,2 \%)$ y en zonas urbanas (88\%) y apenas $45,1 \%$ asume la jefatura de su hogar (columna 1). Sin embargo, quienes se graduaron de universidades públicas (columna 2) tienen una mayor probabilidad de ser bilingües y cabezas de hogar, mientras que las personas graduadas de una universidad privada (columna 3), de ser mujeres, migrantes y laborar en zonas periféricas.

La tabla 4 presenta la distribución de la población graduada por área de estudio. La mayoría (43\%) obtuvo su título en disciplinas sociales, es decir, en ciencias sociales, derecho, comunicación, administración, contabilidad, etc. Además, se observa una mayor concentración de este tipo de 
ESTADÍSTICAS DESCRIPTIVAS PARA LA MUESTRA BALANCEADA. Período: 2011-2017

\begin{tabular}{|c|c|c|c|c|c|}
\hline Universidad & Total & $\begin{array}{c}\text { (2) } \\
\text { Pública }\end{array}$ & $\begin{array}{c}(3) \\
\text { Privada }\end{array}$ & $\begin{array}{l}(4) \\
\text { Prueba* }\end{array}$ & Valor $-\mathrm{p} * *$ \\
\hline Observaciones & 14489 & 5722 & 8767 & & \\
\hline Posee posgrado & $14,9 \%$ & $15,0 \%$ & $14,8 \%$ & 0,4416 & 0,6588 \\
\hline Años de experiencia laboral potencial & 16,7 & 18,0 & 15,8 & 677,6965 & 0,0000 \\
\hline $\begin{array}{l}\text { Porcentaje que desea cambiar trabajo porque no } \\
\text { adquiere experiencia en lo que estudió o no aplica } \\
\text { conocimientos }\end{array}$ & $4,4 \%$ & $4,1 \%$ & $4,7 \%$ & $-120,0000$ & 0,0000 \\
\hline $\begin{array}{l}\text { Porcentaje de personas ocupadas insatisfecha por } \\
\text { desaprovechamiento de competencias }\end{array}$ & $2,7 \%$ & $2,5 \%$ & $2,8 \%$ & $-84,1341$ & 0,0000 \\
\hline Porcentaje con trabajo permanente & $96,5 \%$ & $95,8 \%$ & $97,0 \%$ & 3,4566 & 0,0005 \\
\hline Porcentaje que trabaja en el sector privado & $47,7 \%$ & $46,3 \%$ & $48,7 \%$ & 1,4303 & 0,1526 \\
\hline Porcentaje que trabaja en hogar privado & $0,5 \%$ & $0,5 \%$ & $0,5 \%$ & 0,3085 & 0,7577 \\
\hline \multicolumn{6}{|l|}{$\begin{array}{l}\text { Distribución porcentual por tamaño de la } \\
\text { empresa: }\end{array}$} \\
\hline Microempresa & $12,5 \%$ & $11,4 \%$ & $13,2 \%$ & 3,3166 & 0,0009 \\
\hline Pequeña & $21,7 \%$ & $21,1 \%$ & $22,1 \%$ & 1,4993 & 0,1338 \\
\hline Mediana & $24,4 \%$ & $25,1 \%$ & $24,0 \%$ & $-0,3652$ & 0,7149 \\
\hline Grande & $41,4 \%$ & $42,4 \%$ & $40,7 \%$ & $-3,3586$ & 0,0008 \\
\hline Porcentaje que cuenta con seguro laboral & $93,3 \%$ & $93,1 \%$ & $93,5 \%$ & 1,4447 & 0,1485 \\
\hline Porcentaje que es mujer & $54,5 \%$ & $51,0 \%$ & $56,9 \%$ & 6,8406 & 0,0000 \\
\hline Porcentaje que es bilingüe & $36,2 \%$ & $37,3 \%$ & $35,4 \%$ & $-2,9288$ & 0,0034 \\
\hline Porcentaje que es cabeza del hogar & $45,1 \%$ & $46,9 \%$ & $43,8 \%$ & $-3,6810$ & 0,0002 \\
\hline Porcentaje que tiene pareja & $51,4 \%$ & $51,8 \%$ & $51,1 \%$ & 0,0057 & 0,9954 \\
\hline Porcentaje que es migrante & $6,2 \%$ & $5,0 \%$ & $7,1 \%$ & 4,7849 & 0,0000 \\
\hline Edad promedio & 38,61 & 40,32 & 37,45 & $-14,4950$ & 0,0000 \\
\hline \multicolumn{6}{|l|}{ Distribución porcentual de edad: } \\
\hline 19-29 años & $25,2 \%$ & $22,8 \%$ & $26,8 \%$ & 4,7455 & 0,0000 \\
\hline 30-39 años & $32,4 \%$ & $28,6 \%$ & $35,1 \%$ & 8,5022 & 0,0000 \\
\hline 40-49 años & $22,9 \%$ & $22,4 \%$ & $23,2 \%$ & $-0,5125$ & 0,6083 \\
\hline 50 y más & $19,5 \%$ & $26,3 \%$ & $14,9 \%$ & $-15,1236$ & 0,0000 \\
\hline \multicolumn{6}{|l|}{ Distribución porcentual por región: } \\
\hline Central & $77,2 \%$ & $80,0 \%$ & $75,2 \%$ & $-7,0512$ & 0,0000 \\
\hline Chorotega & $5,6 \%$ & $5,9 \%$ & $5,5 \%$ & $-2,1566$ & 0,0310 \\
\hline Pacífico Central & $3,2 \%$ & $2,8 \%$ & $3,4 \%$ & 2,9148 & 0,0036 \\
\hline Brunca & $5,3 \%$ & $4,2 \%$ & $6,1 \%$ & 5,6938 & 0,0000 \\
\hline Huetar Atlántica & $4,5 \%$ & $3,4 \%$ & $5,2 \%$ & 4,0808 & 0,0000 \\
\hline Huetar Norte & $4,2 \%$ & $3,7 \%$ & $4,6 \%$ & 3,1144 & 0,0018 \\
\hline Zona urbana & $88,0 \%$ & $88,9 \%$ & $87,5 \%$ & 0,2203 & 0,8257 \\
\hline
\end{tabular}

Fuente: Elaboración propia con base en los datos de la ENAHO.

* Para las variables continuas se estima la prueba t para igualdad de medias entre los grupos. Para las variables categóricas se estima la prueba de igualdad de proporción de medias.

** Valor-p asociado a dos colas para la prueba t o prueba de proporcionalidad de medias, según corresponda. 
estudios en las universidades privadas (47,6\%) que en las públicas (36,2\%). Lo anterior es consistente con que las universidades privadas son más pequeñas y brindan una oferta educativa más reducida (PEN, 2019), usualmente concentrada en áreas como esta, pues resulta relativamente más barato ofrecer carreras en estas áreas que en otras que pueden requerir laboratorios y equipo más caro y complejo. Seguidamente, se ubican las personas tituladas en el área de educación (22,5\%), que incluye la educación primaria, secundaria, preescolar, y relacionadas, la cual también supone menores costos para las universidades. En contraste, el porcentaje que estudia áreas relacionadas con matemática, ciencia y computación es reducido $(9,2 \%)$ y similar a quienes estudian ingenierías $(8,4 \%)$; esto muestra que el país no cuenta con una política agresiva para estimular áreas de estudio relacionadas con la innovación tecnológica.

Cabe destacar que, actualmente, las universidades privadas gradúan a casi el doble de personas que las públicas, por lo tanto, aunque cerca de la misma cantidad de estudiantes se matriculen en estas universidades, las privadas gradúan más rápidamente o en mayor volumen. Conforme estas

TABLA 4

ÁREA DE ESTUDIO, SEGÚN TIPO DE INSTITUCIÓN. Período: 2011-2017

\begin{tabular}{lcrrr}
\hline Área de estudio & Total & Pública & Privada & $\begin{array}{c}\text { Porcentaje graduado de } \\
\text { universidad pública }\end{array}$ \\
\hline Total & 14489 & 5722 & 8767 & $40,6 \%$ \\
Educación & $22,5 \%$ & $24,0 \%$ & $21,5 \%$ & $43,3 \%$ \\
Humanidades* & $3,3 \%$ & $3,9 \%$ & $2,9 \%$ & $47,9 \%$ \\
Disciplinas sociales & $43,0 \%$ & $36,2 \%$ & $47,6 \%$ & $34,2 \%$ \\
Ciencias, matemática y computación & $9,2 \%$ & $11,7 \%$ & $7,6 \%$ & $51,3 \%$ \\
Ingenierías, arquitectura y manufacturas** & $8,4 \%$ & $10,4 \%$ & $7,1 \%$ & $49,8 \%$ \\
Agricultura, pesca y ciencias veterinarias & $2,2 \%$ & $4,1 \%$ & $0,9 \%$ & $75,0 \%$ \\
Medicina y estudios relacionados & $9,2 \%$ & $8,1 \%$ & $9,9 \%$ & $35,8 \%$ \\
Servicios & $2,2 \%$ & $1,7 \%$ & $2,5 \%$ & $31,5 \%$ \\
\hline
\end{tabular}

Fuente: Elaboración propia con base en ENAHO.

Nota: *Excluye historia, **incluye producción de alimentos.

personas continúen entrando al mercado laboral, las universidades privadas irán ganando terreno en las diferentes áreas de estudio. De hecho, en la muestra, las personas graduadas de universidades privadas en las disciplinas sociales, medicina y servicios son casi el doble de quienes estudiaron en universidades públicas. Solamente en el área de agricultura, pesca y ciencias veterinarias, el porcentaje observado en la muestra de personas graduadas de las instituciones públicas es significativamente superior al de las privadas. Es importante aclarar que en el área de humanidades se incluyen los estudios de inglés, religión, bellas artes y deportes y que en el área de servicios se toman en cuenta las carreras de hotelería, transporte, seguridad y cuido.

Por otra parte, si se analiza la media del salario bruto real por hora del trabajo principal, las personas graduadas de posgrados tienen salarios significativamente mayores $(71 \%$ más) a las graduadas de grado, así como las graduadas de universidades estatales tienen salarios significativamente mayores (7\%) a las graduadas de universidades privadas. Tal información da señales del peso que tiene la universidad pública sobre los rendimientos de la educación y también que, a pesar de que se esté tomando una población con estudios universitarios en su totalidad, los retornos de los posgrados son significativos, i.e., se observa rendimientos crecientes respecto al nivel educativo. 
TABLA 5

SALARIO BRUTO REAL POR HORA MEDIO EN EL EMPLEO PRINCIPAL, SEGÚN GRADO ACADÉMICO Y TIPO DE INSTITUCIÓN. Período: 2011-2017

\begin{tabular}{lrrr}
\hline $\begin{array}{l}\text { Salario bruto real en el empleo } \\
\text { principal }\end{array}$ & Media & $\begin{array}{c}\text { Error } \\
\text { estándar }\end{array}$ & Observaciones \\
\hline Población & 5241,8 & 33,0 & 14489 \\
\hline Grado académico: & 4757,2 & 30,0 & 12420 \\
$\quad$ Grado & 8150,7 & 127,3 & 2069 \\
$\quad$ Posgrado & 5460,1 & 53,9 & 5722 \\
Tipo de institución: & 5099,3 & 41,6 & 8767 \\
$\quad$ Pública & & & 4914 \\
$\quad$ Privada & 4973,3 & 50,8 & 7506 \\
Grado académico y tipo de institución: & 4597,0 & 35,9 & 808 \\
$\quad \begin{array}{l}\text { Grado y pública } \\
\text { Grado y privada }\end{array}$ & 8322,7 & 186,3 & 1261 \\
\hline $\begin{array}{l}\text { Posgrado y pública } \\
\text { Posgrado y privada }\end{array}$ & 8005,9 & 168,8 & \\
\hline
\end{tabular}

Fuente: Elaboración propia con datos de la ENAHO (2011-2017).

Nota: Todas las medias son significativamente diferentes entre ellas.

\section{Metodología}

En aras de encontrar si efectivamente hay diferencias significativas entre la educación superior pública y privada se llevaron a cabo dos análisis. El primero es descriptivo y en él se estimaron las tasas de emparejamiento correcto entre las personas trabajadoras y sus años de escolaridad, con el fin de cuantificar el nivel de sobreeducación en personas graduadas de universidades públicas y privadas. El segundo análisis estudia si los rendimientos de la educación sobre el salario de las personas graduadas difieren según el tipo de institución, así como el efecto de la sobreeducación sobre el salario. Para ello, se utilizó una muestra de personas graduadas de la educación superior (en adelante personas graduadas) de la Encuesta Nacional de Hogares para el período 2011-2017. Esta es una encuesta anual de representación nacional que recopila información de más de diez mil hogares sobre su composición y condiciones de vida. Además, recolecta información individual sobre sus miembros, incluyendo sus características sociodemográficas, educativas y de empleo. La ENAHO es de corte transversal, de forma que los hogares encuestados varían año con año.

\section{Metodología para la estimación del tipo de emparejamiento laboral}

Existen tres métodos para estimar la sobreeducación: el normativo u objetivo, el subjetivo y el estadístico (Alpin, Shackleton, \& Walsh, 1998; Dolton \& Silles, 2001; Quintini, 2011). En el primero, se utiliza una escala objetiva para clasificar los requerimientos educativos de cada ocupación, tal como la escala de competencias ISCO-08 (ILO, 2012), y luego se compara el nivel de competencia de la persona con el requerido para su ocupación. Si el nivel de competencia observado supera al requerido, se dice que la persona se encuentra sobrecalificada. En el método subjetivo se le 
pregunta a la persona si considera que su nivel de educación está acorde con su puesto de trabajo. Finalmente, el método estadístico consiste en comparar los años de escolaridad de la persona con el promedio para la ocupación. Si la persona se desvía más de un cierto valor, medido usualmente en términos de una desviación estándar, se dice que se encuentra sobreeducada.

Existen ventajas y desventajas en utilizar cada método. El normativo ofrece la mayor objetividad y permite establecer criterios uniformes de comparación. Esto es particularmente útil si se busca realizar comparaciones entre países o con respecto a una norma estandarizada, asî como estudiar toda la población ocupada, pero también puede resultar rígido si se desea capturar la evolución de los requerimientos de competencias asociados a una ocupación o analizar características de un caso particular. El método subjetivo puede resultar menos costoso, pero la calidad de la información está sujeta al criterio individual de quienes responden. Y, el método estadístico refleja la evolución y particularidades de cada mercado, pues su punto de referencia es la media observada para la muestra analizada, pero dificulta la estandarización y comparación entre países.

La ENAHO permite la estimación de la sobrecalificación y sobreeducación utilizando tanto el método normativo como el estadístico. Debido a que una de las diferencias más importantes observadas entre los programas de universidades públicas y privadas en Costa Rica es su longitud, se optó por utilizar el método estadístico para realizar el análisis, ya que el método normativo no permitiría captar variaciones asociadas a estas particularidades de la educación superior costarricense. Por ejemplo, tómese el caso de los programas de psicología de la primera universidad pública y la primera universidad privada en la clasificación Webometrics (Cybermetrics Lab, 2019). En la Universidad de Costa Rica (2017), el programa de licenciatura en psicología está programado para diez semestres, i.e., cinco años de educación superior, mientras que en la Universidad Latina de Costa Rica (2019) el mismo grado académico se puede obtener en 10 cuatrimestres, o sea, algo más de 3 años. Supóngase, entonces dos personas con licenciatura, donde la primera cursó un programa de cinco años mientras que la segunda cursó uno de tres. Si ambas laboran en un puesto donde este grado académico es un requisito, ambas aparecerán como adecuadamente empatadas con su trabajo bajo el método normativo, mientras que el método estadístico podría identificar si una persona con un bachillerato de 5 años se encuentra sobreeducada en comparación con la media ocupacional. Para el caso costarricense, esta diferencia podría ser significativa, ya que las personas graduadas de universidades públicas reportan más años de escolaridad que quienes se graduaron de privadas y este último grupo entra con mayor rapidez y en mayor número al mercado laboral, por lo que tiene un mayor peso en la determinación del comportamiento medio de una ocupación.

Siguiendo a Chevalier (2003), se distingue entre aquellas personas que se encuentran genuinamente sobreeducadas en el trabajo y quienes solo lo están aparentemente, a partir de su satisfacción laboral. La relación trabajo-educación-habilidades es la que indica si las personas están genuina o aparentemente sobreeducadas. Utilizando las preguntas sobre satisfacción laboral $y$ las motivaciones para desear cambiar de trabajo, se clasifica como genuinamente sobreeducadas a aquellas personas con sobreeducación y que también deseen cambiar de trabajo porque en el actual no aprovechan su conocimiento o estudios o no pueden adquirir o aumentar su experiencia en lo que quieren, así como a quienes cuentan con sobreeducación y reportan insatisfacción laboral por desaprovechamiento de competencias. Por el contrario, aquellas personas que se hallen sobreeducadas, pero no deseen cambiar de trabajo ni reporten insatisfacción laboral, se catalogan como aparentemente sobreeducadas. 


\section{Metodología para la estimación de las ecuaciones salariales}

Los retornos de la educación se estimaron utilizando ecuaciones de Mincer (1974). Esta es la metodología mediante la cual se acostumbra estimar los salarios ${ }^{7}$. Se calcula el logaritmo natural del salario real por hora $(w)$ mediante mínimos cuadrados ordinarios en función de una matriz X de variables asociadas al nivel y la calidad del capital humano, características del empleo y la empresa, características sociodemográficas y un vector de errores $\varepsilon$. Las variables relacionadas con la calidad del capital humano son las tres variables de interés: una dicotómica que indica si se asistió a una universidad pública y dos categóricas para el área de estudio y tipo de emparejamiento laboral. Si la calidad difiere según el tipo del centro educativo, se observará un coeficiente significativo asociado a la variable dicotómica que indica si el centro educativo es público. Las áreas de estudio se asocian a diferentes tipos de conocimiento, con sus respectivos retornos (Chevalier, 2011; Machin \& Puhani, 2002; 2005). Por último, se toma en cuenta el tipo de emparejamiento laboral de la persona, no solo para estudiar el efecto de la sobreeducación sobre los salarios, sino también para separar este efecto de aquel de la educación en general. Por su parte, las variables asociadas al nivel del capital humano incluyen una variable dicotómica que indica si la persona cuenta con posgrado y las variables continuas para los años de experiencia laboral potencial y su cuadrado. La experiencia laboral potencial se calcula como la edad menos los años de educación y los seis años de preescolar.

Se estimaron tres modelos y, a su vez, se calculó cada uno de ellos separando la muestra por sexo, ya que, a pesar de que el enfoque de la investigación es respecto a la diferencia entre la calidad de la educación superior pública y privada, estructuralmente las mujeres tienen salarios menores que los hombres (Becker, 1971; Blau, 1999; Blau \& Kahn, 2007; Goldin, 2002; Mumford \& Smith, 2009; Oaxaca, 1973; Rosenfeld, 1979; Stotsky, 2006). En el primer modelo, se incluyeron las variables relacionadas al capital humano. En el segundo, se controló por características del empleo y la empresa. Estas son cuatro variables dicotómicas indicando si la persona cuenta con un trabajo permanente, labora en el sector privado, en un hogar privado y si cuenta con seguro laboral y una variable categórica para el tamaño de la empresa. En el tercer modelo, se controló, además, por seis variables sociodemográficas: cinco dicotómicas, indicando si la persona es bilingüe, cabeza del hogar o migrante, si tiene pareja o si vive en zona urbana y una variable categórica para la región. Se presentan los tres modelos para analizar la robustez de las estimaciones. Antes de estimar las regresiones, se verificó que las variables no se encontraran correlacionadas ${ }^{8}$.

Debido a que la decisión del salario es simultánea a la decisión sobre el tipo de emparejamiento laboral, en todos los modelos se realizó un ajuste de Heckman (1976) incluyendo las variables correspondientes a la inversa de la razón de Mills asociadas a las probabilidades de aparente y genuina sobreeducación de un modelo logit multinomial ${ }^{9}$, conforme la metodología

$7 \quad$ No se estimaron las ecuaciones de salarios con otras metodologías. Como se menciona más adelante, se consideró que la estimación de salarios propuesta por Dolton \& Silles (2001), que utiliza ecuaciones de Mincer, era la mejor metodología, ya que permite corregir la simultaneidad de las decisiones del salario y tipo de emparejamiento al estimar salarios utilizando bases de datos de corte transversal.

8 En ningún caso la correlación de Pearson supera el valor de 0,37; por lo que se descarta la correlación fuerte entre variables. Además, si bien se tienen observaciones para diferentes años, esto no supone riesgo de autocorrelación serial, debido a que no se cuenta con ningún tipo de serie de tiempo.

9 La regresión logit multinomial se estimó considerando la ocupación, variables asociadas a características del empleo (empleo permanente, tiempo parcial, tamaño de la empresa, sector), características sociodemográficas (si posee estudios de posgrado, área de estudio, jefatura del hogar, si tiene pareja, si es migrante, si es mujer, la cantidad de personas en edad preescolar en el hogar, el tamaño del hogar, cantidad de personas mayores de quince años que se encuentran desempleadas o inactivas en el hogar, edad, edad al cuadrado, región y si vive en zona urbana) y la correspondiente corrección por autoselección en la participación laboral. Debido a limitaciones de espacio, estas estimaciones no se reportan, pero están disponibles bajo solicitud. 
de Dolton \& Silles $(2001)^{10}$ y correcciones similares en Mayston \& Yang (2008) y Blanco (2019). Es decir, previo a estimar las ecuaciones salariales, debe calcularse una estimación del tipo de emparejamiento (E) con un modelo latente logit multinomial de la siguiente forma:

$$
E=\left\{\begin{array}{l}
0 \text { si } E^{*}<\mu_{0} \\
1 \text { si } E^{*}<\mu_{1} \\
2 \text { si } E^{*}<\mu_{2}
\end{array}\right.
$$

donde el tipo de emparejamiento identifica si la persona se encuentra adecuadamente empatada con su trabajo (categoría 0), aparentemente sobreeducada (categoría 1) o genuinamente sobreeducada (categoría 2). A partir de cada una de estas ecuaciones, se puede obtener el inverso de la razón de Mills ( $)$ asociado a la probabilidad de que una persona esté aparente o genuinamente sobreeducada. Estos valores son posteriormente introducidos en la ecuación de salarios como correcciones de ajuste por endogeneidad. Si los coeficientes asociados a dichas variables son significativamente diferentes de cero, la corrección por endogeneidad ${ }^{11}$ es entonces pertinente. Por tanto, el modelo a estimar está dado de la siguiente manera:

$$
\operatorname{In}(w)=\beta X+\delta \lambda+\varepsilon
$$

A partir del tercer modelo, se hizo un análisis por grupos de edad con el fin de someter a prueba si la significancia del tipo de universidad en la determinación del salario disminuye conforme avanza la edad para las diferentes cohortes. Esto sería así si, al inicio de la vida laboral, lo que más tiene peso es el título académico, pero, conforme transcurre el tiempo, este deja de tener tanta relevancia y, en su lugar, la experiencia laboral o la reputación individual son más relevantes al negociar el salario. Por ello, se desea analizar, separando por cohortes, qué tanto peso tiene un título en educación superior pública en relación con la educación superior privada para cada etapa de la vida laboral. Para esto, la población se dividió en los siguientes cuatro grupos de edad: de 19 a 29 años, de 30 a 39, de 40 a 49 años y las personas de 50 años o más.

Por último, se reestima la ecuación salarial en (2) sustituyendo las variables de tipo de institución y área de estudio por una variable categórica que refleja las interacciones entre estas. Entonces, se tendrá una diferenciación en los títulos, lo que sirve para analizar el efecto que tiene cada una de las áreas de estudio de cada tipo de institución sobre el salario. De esta forma, se podrá analizar si titularse en un área es más rentable en el sector público o el privado.

10 También se realizaron pruebas de corrección por autoselección asociadas a la decisión de participación laboral (Heckman, 1976), pero no se encontró evidencia de autoselección, por lo que se reportan los resultados sin esta corrección.

11 Dado que el tipo de emparejamiento se estima mediante una logit multinomial, no es posible realizar la prueba de Hausman para endogeneidad, pues esta requiere que las ecuaciones del sistema sean lineales. La endogeneidad entre el tipo de emparejamiento y el salario se asume teóricamente, debido a la simultaneidad de las decisiones, pues al aceptar un empleo particular se está decidiendo el salario y el tipo de emparejamiento. La forma en que se corrige esta endogeneidad teórica es mediante la inclusión de las variables correspondientes a la inversa de la razón de Mills mencionadas anteriormente. Tampoco puede realizarse una prueba de endogeneidad de Hausman entre el salario y la decisión de estudiar un posgrado, pues no se cuenta con buenos instrumentos para medir la decisión de estudiar un posgrado y dicha variable es dicotómica, no lineal. No se contempló sustituir esta variable por los años de educación, ya que los programas de estudio entre las diferentes disciplinas y tipos de institución varían en longitud y resulta de mayor interés poder identificar el grado académico. 


\section{RESULTADOS}

\section{Emparejamiento laboral}

La tabla 6 presenta los resultados obtenidos para el tipo de emparejamiento laboral y muestra que la sobreeducación genuina es prácticamente inexistente para Costa Rica. Esto es esperable, ya que el mercado laboral costarricense se caracteriza por su bajo capital humano: únicamente 15\% de la población mayor de edad cuenta con estudios superiores, por lo que es de esperar que la mayoría de las personas graduadas sea capaz de encontrar un puesto de trabajo acorde con sus habilidades. Además, aunque las personas graduadas de educación superior privada tienen una tasa de genuina sobreeducación mayor que las de graduadas de públicas, esta diferencia es menor a un punto porcentual, lo que resulta casi despreciable.

En realidad, el dato más interesante de la tabla 6 es el de la tasa de sobreeducación aparente, pues es sumamente alta en ambos tipos de institución. Más de la mitad de la población graduada de instituciones públicas es aparentemente sobreeducada, así como un $42,4 \%$ de las privadas. Esto podría interpretarse de diferentes maneras: en primer lugar, podría sugerir una inflación en la titulación, i.e., al ampliarse la matrícula en las universidades, estas empiezan a admitir a personas menos hábiles, por lo que los títulos universitarios se vuelven menos eficientes en identificar a la población de mayor habilidad y esto obliga a quienes son efectivamente más hábiles a capacitarse más para obtener los mismos puestos de trabajo.

Una segunda interpretación refiere a la escasez de personas con niveles de competencias técnicas y profesionales de nivel intermedio: la escasez de ofertas académicas en el país para desarrollar competencias de nivel intermedio podría estar induciendo a personas con habilidades medias a sobre invertir en educación para obtener títulos universitarios, a pesar de que luego van a ir al mercado laboral a ocupar puestos de nivel intermedio. Esto apuntaría a la necesidad de reformar la oferta educativa para ofrecer posibilidades de capacitación en el desarrollo de competencias de nivel intermedio. Lo anterior supondría un mecanismo más eficiente para generar los emparejamientos laborales adecuados. Además, es probable que los títulos que se están ofreciendo en las universidades no coincidan con lo que las empresas están demandando. Resultaría interesante estudiar el porqué de tales resultados en estudios posteriores. 


\section{TABLA 6 \\ TIPO DE EMPAREJAMIENTO LABORAL, SEGÚN GRADO ACADÉMICO Y TIPO DE INSTITUCIÓN. Período: 2011-2017}

\begin{tabular}{|c|c|c|c|}
\hline & $\begin{array}{r}\text { Total } \\
(\%)\end{array}$ & $\begin{array}{c}\text { Pública } \\
(\%)\end{array}$ & $\begin{array}{c}\text { Privada } \\
(\%)\end{array}$ \\
\hline \multicolumn{4}{|l|}{ Total: } \\
\hline Adecuada educación & 50,5 & 44,7 & 54,6 \\
\hline Aparente sobreeducación & 46,5 & 52,6 & 42,4 \\
\hline Genuina sobreeducación & 3,0 & 2,8 & 3,1 \\
\hline \multicolumn{4}{|l|}{ Grado: } \\
\hline Adecuada educación & 55,9 & 49,5 & 60,3 \\
\hline Aparente sobreeducación & 40,9 & 47,4 & 36,5 \\
\hline Genuina sobreeducación & 3,2 & 3,1 & 3,2 \\
\hline \multicolumn{4}{|l|}{ Posgrado: } \\
\hline Adecuada educación & 19,8 & 17,5 & 21,4 \\
\hline Aparente sobreeducación & 78,6 & 81,7 & 76,4 \\
\hline Genuina sobreeducación & 1,7 & 0,8 & 2,3 \\
\hline
\end{tabular}

Fuente: Elaboración propia con base en ENAHO.

Nota: Todas las medias son significativamente diferentes entre ellas.

Por último, una tercera interpretación se relaciona con la construcción misma del indicador: recuérdese que no se utilizó el método normativo sino el estadístico a partir de los años de educación, de forma que estas altas tasas de aparente sobreeducación reflejan que existe un porcentaje importante de personas graduadas con más años de educación acumulados relativo al promedio de su ocupación. Las universidades brindan una diversidad de programas académicos diferentes en extensión, pero si la mayoría de ellas ofrecen alternativas cortas, por ejemplo, de tres años, aquellas con programas más largos $-y$ esto afecta particularmente a las universidades públicas- estarían sobreeducando a sus estudiantes para el mercado interno. Esto plantea una seria disyuntiva en cuanto a las políticas educativas y remite a las diferencias observadas en la clasificación de las universidades: si un grupo selecto de universidades aspira a formar estudiantes con niveles internacionales $y$ a ascender en las clasificaciones internacionales -piénsese, por ejemplo, en el caso de las universidades públicas-, pero el mercado laboral interno está siendo dirigido por políticas que más bien reducen la calidad y extensión de los programas académicos, las personas graduadas del primer grupo se verán perjudicadas si se preparan para trabajar en el mercado interno, porque estarán realizando una inversión excesiva para optar por puestos diseñados para programas inferiores. De ser este el caso, cabría plantearse la posibilidad de competir con las universidades del segundo grupo a través de diplomados, i.e., programas cortos similares a los ofrecidos por este segundo grupo, con el fin de señalizarle a las empresas las diferencias existentes entre unos y otros. También podría cederse a la presión del mercado interno y adecuar los programas para homologarlos hacia la media, pero esto supondría sacrificar las aspiraciones de internacionalización y ajustarse hacia un equilibrio inferior.

Analizando únicamente la población con grado universitario, las tasas de aparente sobreeducación disminuyen en todos los casos. En las personas graduadas de universidad privada, los números alcanzan un porcentaje cercano a un tercio de la población, mientras que aquellas provenientes de universidades públicas llegan a poco menos del cincuenta por ciento. Sin embargo, 
las tasas siguen siendo muy altas. Respecto a la población graduada de posgrado, los datos son más alarmantes y proveen evidencia a favor de la hipótesis de la inflación educativa. Esta es una clara señal de discordancia entre la oferta educativa y la demanda laboral y alerta sobre la necesidad de coordinar dicha oferta educativa para suplir las necesidades del mercado.

\section{Ecuaciones salariales}

La tabla 7 muestra los resultados de la ecuación de Mincer para las variables de interés. Como se mencionó anteriormente, la muestra consiste en 14489 observaciones, de las cuales 8002 corresponde a mujeres y 6 487, a hombres. En todos los casos, el valor del estadístico F muestra que los modelos estimados difieren de un modelo sin regresores. Además, el $\mathrm{R}^{2}$ muestra que entre $23,8 \%$ (columna 2) y 34,1\% (columna 8) de la variancia observada en el logaritmo del salario por hora es explicada por el modelo. Este valor es consistente con el $\mathrm{R}^{2}$ obtenido por Chevalier \& Lindley (2009) de 24\%, aunque es algo inferior al de Chevalier (2003), donde oscila entre $32 \%$ y 44\%. No obstante, Chevalier (2003) posee como ventaja que cuenta con bases de datos longitudinales que le permiten capturar habilidades no observadas y que no pueden ser estimadas con bases de datos de corte transversal.

Las columnas 1, 2 y 3 corresponden al primer modelo, el cual no incluye variables de control. Se puede observar que haber realizado estudios de posgrado ocasiona que el salario sea en promedio $110,2 \%$ mayor al de quienes solo se quedaron con estudios de grado ${ }^{12}$, a una significancia del $1 \%$ (columna 1). Esta ventaja es mayor para los hombres $(111,9 \%)$ que para las mujeres $(105,9 \%)$ (columnas 3 y 2 , respectivamente). Además, se encuentra que, si se asiste a la educación superior pública, el salario de los hombres será 6,2\% mayor al de quienes asistieron a educación superior privada (columna 3), mientras que, las mujeres graduadas de educación pública ganan, en promedio, $8,3 \%$ (columna 2) más que las mujeres graduadas de educación privada, a un nivel de confianza del 99\%. Por tanto, existe un premio por estudiar en una institución pública para ambos sexos.

Además, se puede notar que, tanto para el modelo agregado (columna 1) como el masculino (columna 3), haber estudiado humanidades se relaciona con un salario $14,0 \%$ y 22,2\% menor al de quienes estudiaron educación. Asimismo, las áreas relacionadas con servicios no muestran diferencias significativas con respecto a la categoría de referencia. Las restantes áreas ganan, en promedio, un salario por hora superior al de alguien que haya estudiado educación en el modelo agregado (columna 1), aunque esto varía según el sexo. Considerando a las mujeres únicamente, se encuentra que aquellas que estudiaron en el área de agricultura, pesca y ciencias veterinarias son quienes reciben el mayor salario promedio, con un premio de $20,2 \%$ respecto a las mujeres en la categoría de referencia, y si lo hicieron en ciencias, matemática o computación, el premio es de 15,3\% más, mientras que en ingeniería, arquitectura o manufactura es de 13,9\% (columna 2). Para los hombres (columna 3), las áreas que, en promedio, ganan más comparado con aquellos hombres que estudiaron educación son: medicina y estudios relacionados, con un premio de 22,5\%, ingenierías, arquitectura y manufacturas, con $16,5 \%, y$ ciencias, matemática y computación, con $14,1 \%$. Estudiar medicina (agricultura, pesca y ciencias veterinarias) no reporta un mayor salario para las mujeres (hombres) relativo a sus pares en educación.

Conforme a la teoría, aquellas personas sobreeducadas reciben un salario promedio inferior al de quienes cuentan con su mismo nivel de educación y se hallan bien emparejadas. Así, quienes tienen una aparente sobreeducación reportan un salario $26 \%$ inferior al de quienes no tienen sobreeducación (columna 1) y esto afecta más a los hombres (-28\%) (columna 3) que a las mujeres (-24\%) (columna 2). Similarmente, las personas genuinamente sobreeducadas ganan, en promedio,

12 Recuérdese que los coeficientes de las variables categóricas deben interpretarse con el antilogaritmo menos 1. En este caso, $110,2 \%=e^{0.743}-1$. 
46,4\% menos (columna 1) que la categoría de referencia y, nuevamente, los hombres son los más afectados (columna 3). También conforme a la teoría, el salario se asocia positiva y decrecientemente con la experiencia laboral. Finalmente, la decisión salarial sí parece ser simultánea con la decisión de tomar un trabajo para el cual se tiene una aparente sobreeducación, más no con respecto a la genuina sobreeducación.

Las columnas 4, 5 y 6 utilizan las características de la empresa como variables de control, mientras que las columnas 7, 8 y 9 controlan tanto por las características de la empresa como las sociodemográficas. Como es de esperarse, el $\mathrm{R}^{2}$ mejora con la inclusión de las variables de control. Aun incluyendo estas variables, haberse graduado de una universidad pública se relaciona positiva y significativamente (al 1\%) con el salario (columnas de 4 a 9), lo que sugiere que efectivamente podrían existir diferencias en la calidad de la educación universitaria en el país. La persistencia de la significancia muestra la robustez de esta variable sobre el salario. El premio por obtener un título en educación superior pública es de 4,7\% para los hombres con respecto a aquellos con educación superior privada (columnas 6 y 9), mientras que el de las mujeres oscila entre 5,8\% (columna 8) y $6,7 \%$ (columna 5), ambos con una confianza del 99\%. Con los controles, los premios de las mujeres (columna 8) relacionados a las áreas de estudio identificados en el modelo sin controles (columna 2) continúan siendo significativos, pero el premio asociado a las ingenierías y similares $(13,9 \%)$ ahora supera al de ciencias, matemática y computación (7,9\%). Además, las mujeres graduadas de programas en servicio ganan, en promedio, 11,3\% (columna 8) menos que sus pares en educación. Para los hombres (columna 9), estudiar medicina continúa siendo el área de estudio más rentable $(12,4 \%)$, seguido de las ciencias, matemáticas y computación, cuyo premio relativo a la categoría de referencia (11,6\%) ahora supera al de ingenierías y similares (8,7\%). En las columnas 4 a 9 , el castigo por la aparente sobreeducación sigue siendo menor que el asociado a la genuina sobreeducación, conforme a lo predicho por la teoría.

\section{Ecuaciones salariales por cohorte}

La tabla 8 muestra las estimaciones de los coeficientes asociados a las regresiones por grupo de edad. Al igual que antes, estos modelos son diferentes a uno sin regresores y logran explicar entre $24,5 \%$ y $30 \%$ de la variancia observada en el logaritmo de los salarios por hora. Resulta interesante que el coeficiente relacionado con el posgrado es mayor para la cohorte de hombres entre 40 y 49 años, quienes ganan 73,5\% más que sus homólogos con grado universitario (columna 9), mientras que, en las mujeres, el mayor premio se registra en la cohorte con edades entre 30 y 39 años $(72,5 \%)$ (columna 5), ambos al 1\% de significancia.

Tal y como se predijo, tener un título de una institución pública es significativo y positivo en los primeros años laborales para el modelo agregado y los hombres, pero no en los años posteriores ${ }^{13}$. Para las mujeres, el coeficiente asociado a graduarse de una institución pública es significativo para las cohortes con edades entre 30 y 49 años (columnas 5 y 8), mas no asî para la cohorte más joven. Esto puede deberse a que las mujeres con edades entre 19 y 29 años se encuentran en la etapa de mayor reproducción y, por tanto, presentan más intermitencia en el trabajo. Después de los 30 años, las mujeres profesionales podrían tener un mayor apego al mercado laboral $y$ es en este momento cuando se observa la diferencia en el ingreso asociada al tipo de universidad donde se estudió. Para los hombres, estudiar en una universidad pública se

13 Intuitivamente, esto sugiere que la experiencia laboral se vuelve más relevante con la edad. Sin embargo, otra interpretación podría ser que las primeras universidades privadas sean más similares a las universidades públicas y que, por tanto, no se observe diferencias en la cohorte de mayor edad. No obstante, los datos sobre la calidad educativa, medida a través de Webometrics (Cybermetrics Lab, 2019) no respaldarían esta hipótesis. 
asocia con un mayor salario para las cohortes hasta con 39 años, al nivel de confianza estándar (columnas 3 y 6). El mayor efecto del título de universidad pública es alcanzado por los hombres entre los 30 y 39 años, quienes reportan un salario 9,5\% mayor que el de los hombres con títulos de universidades privadas (columna 6). Para las mujeres, este pico se da entre los 40 y 49 años, con un efecto de $8,1 \%$ respecto al salario de las mujeres graduadas de universidades privadas (columna 8). Para ambos sexos, el premio por estudiar en una universidad pública podría extenderse alrededor de los primeros 20 años de vida laboral estable, aunque para las mujeres este efecto se observa en forma retardada. El coeficiente asociado a los años de experiencia laboral potencial únicamente es significativo para la cohorte más joven (columnas 1 a 3), no porque la experiencia como tal no sea relevante al explicar el salario, sino más bien porque esta variable se construye en relación con la edad y, al estimarse las regresiones por cohortes, los años de experiencia potencial son similares $y$ pierden, por tanto, variabilidad.

Debido a que se controla por las variables sociodemográficas y del empleo, la mayoría de los coeficientes asociados al área de estudio pierden su significancia para las cohortes femeninas. Por ejemplo, el premio que se observa asociado a las ingenierías en la cohorte más joven (columna 2) desaparece para las cohortes con 30 años o más. Para los hombres sí se observa robustez en el premio asociado por estudiar ciencias, matemática y computación (columnas 3, 9 y 12), así como el castigo asociado a las humanidades (columnas 3, 6, 9 y 12). Para los hombres, además, se observa que el castigo por la aparente y genuina sobreeducación es creciente conforme aumenta la edad de las cohortes. Ahora bien, es de esperar que, con el tiempo, las personas sobreeducadas logren moverse a un puesto de trabajo más acorde con sus habilidades. Si, a pesar del tiempo, ellas persisten en esta condición, probablemente lo hagan porque, efectivamente, poseen menores habilidades que sus pares y es lógico que estas diferencias se amplifiquen. En contraste, las mujeres solo presentan este comportamiento cuando están genuinamente sobreeducadas, mas no cuando están aparentemente sobreeducadas. 


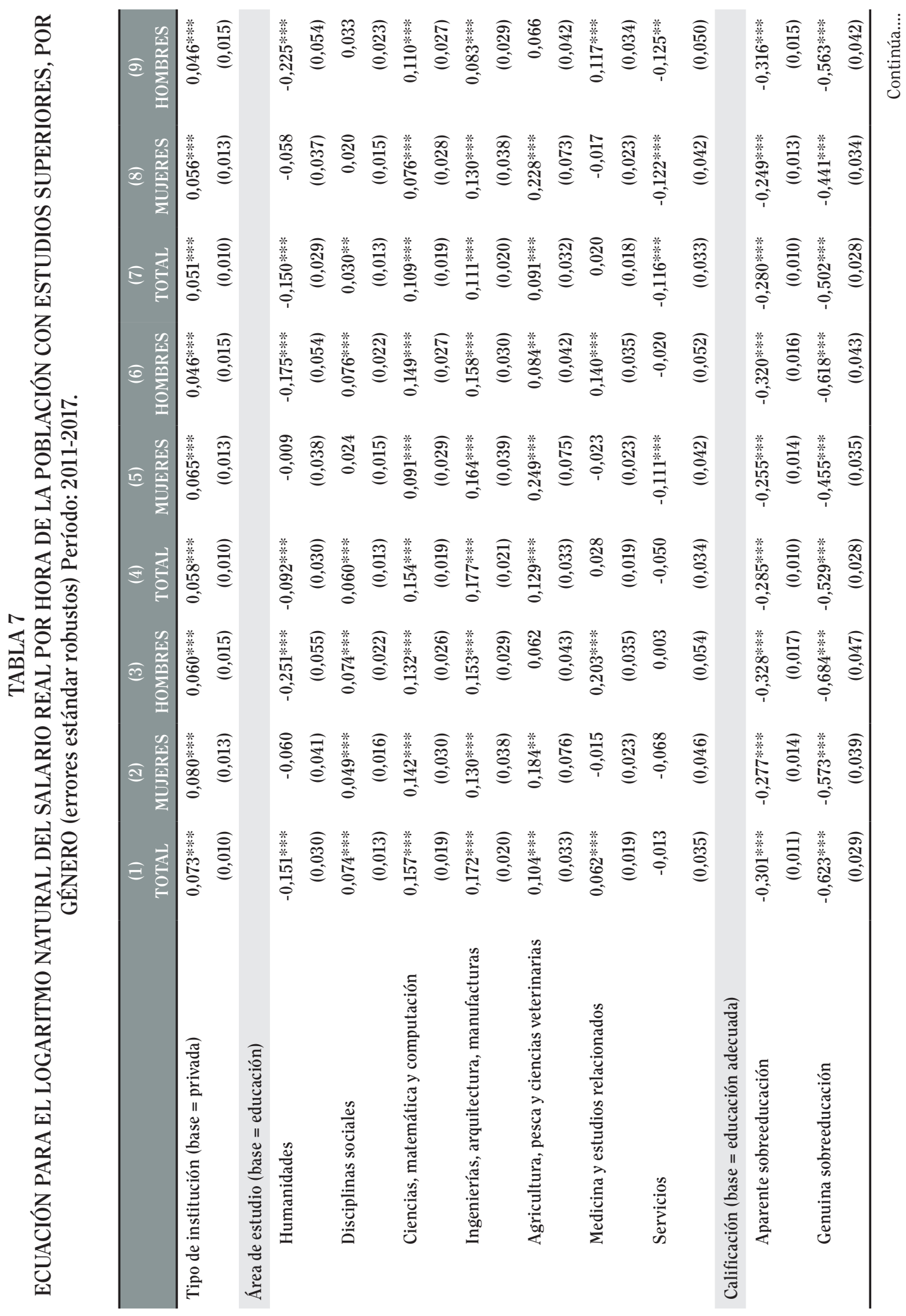




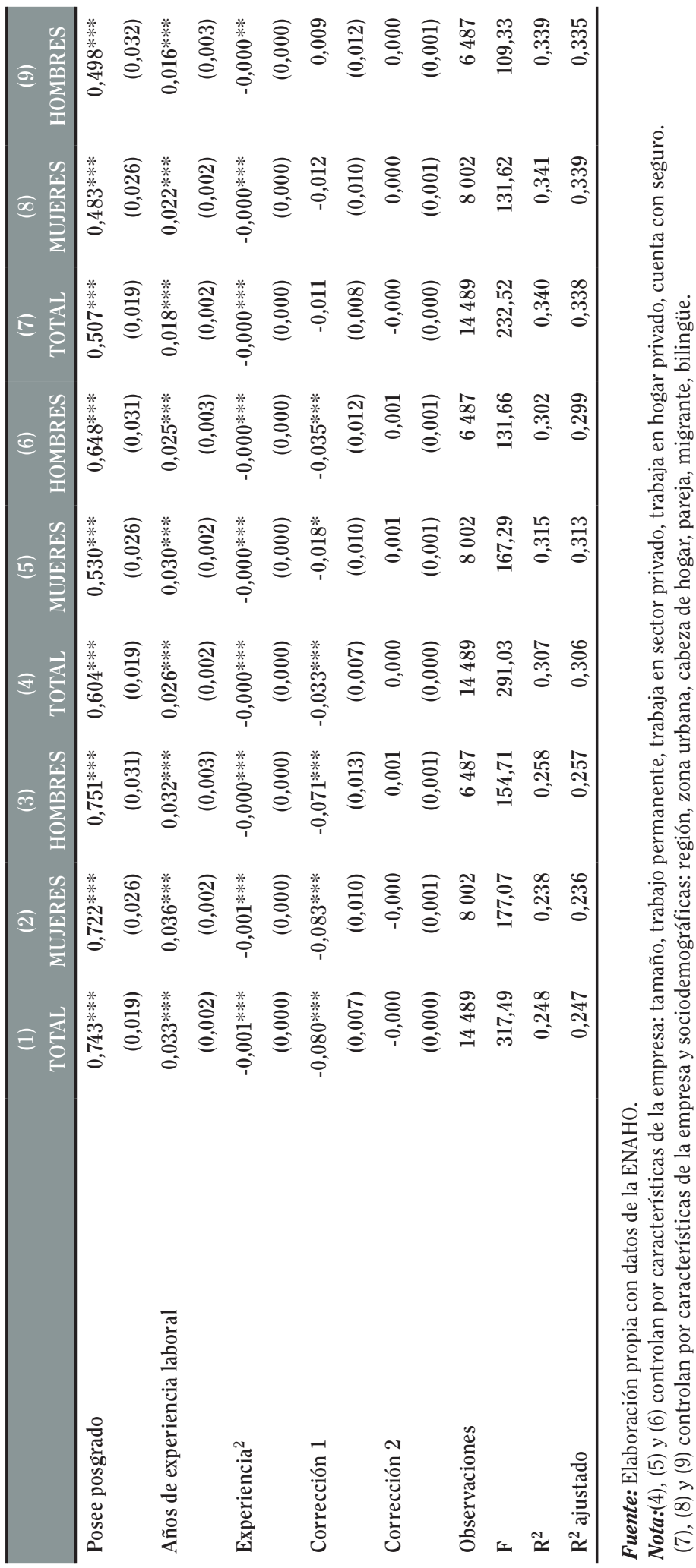




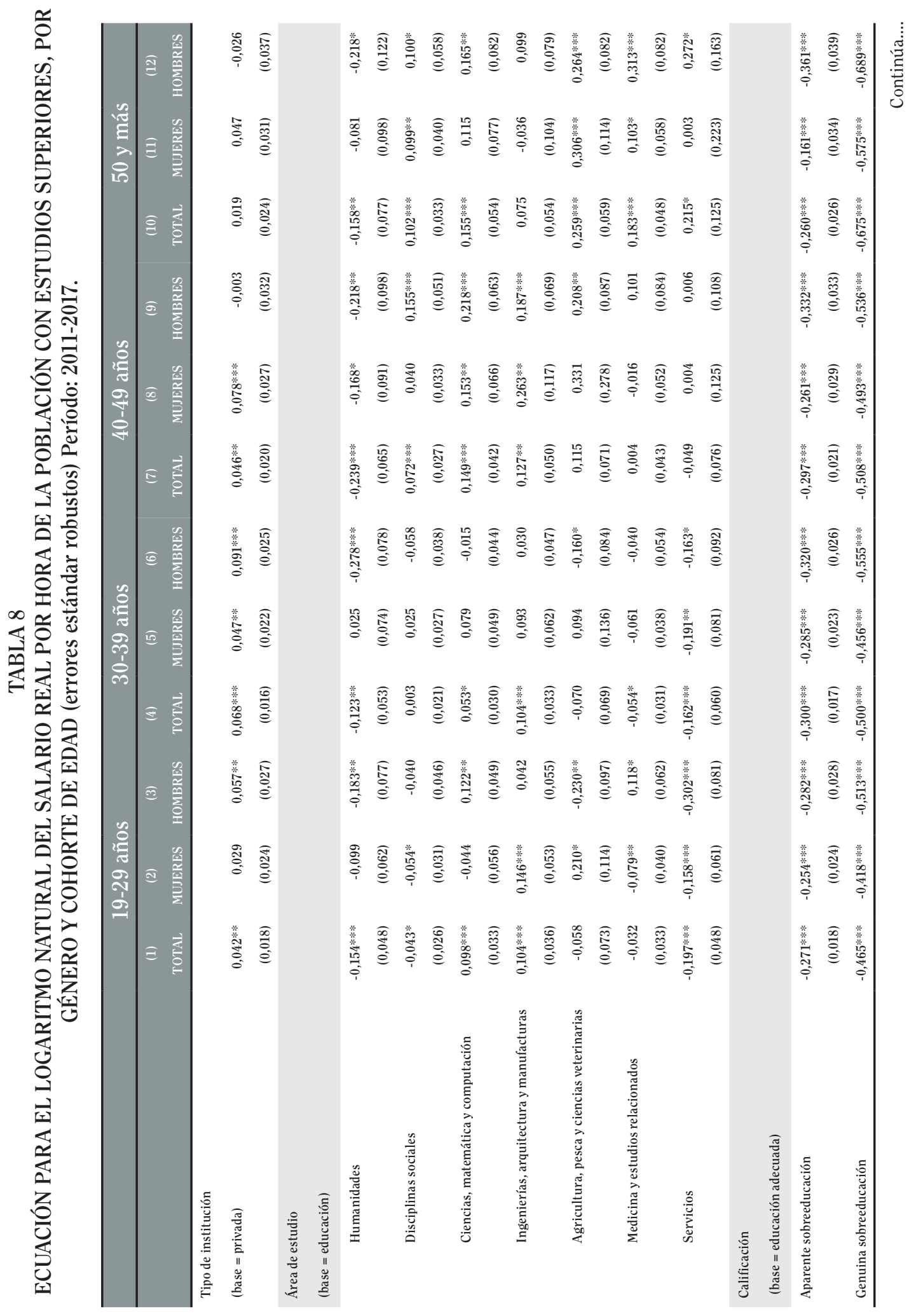




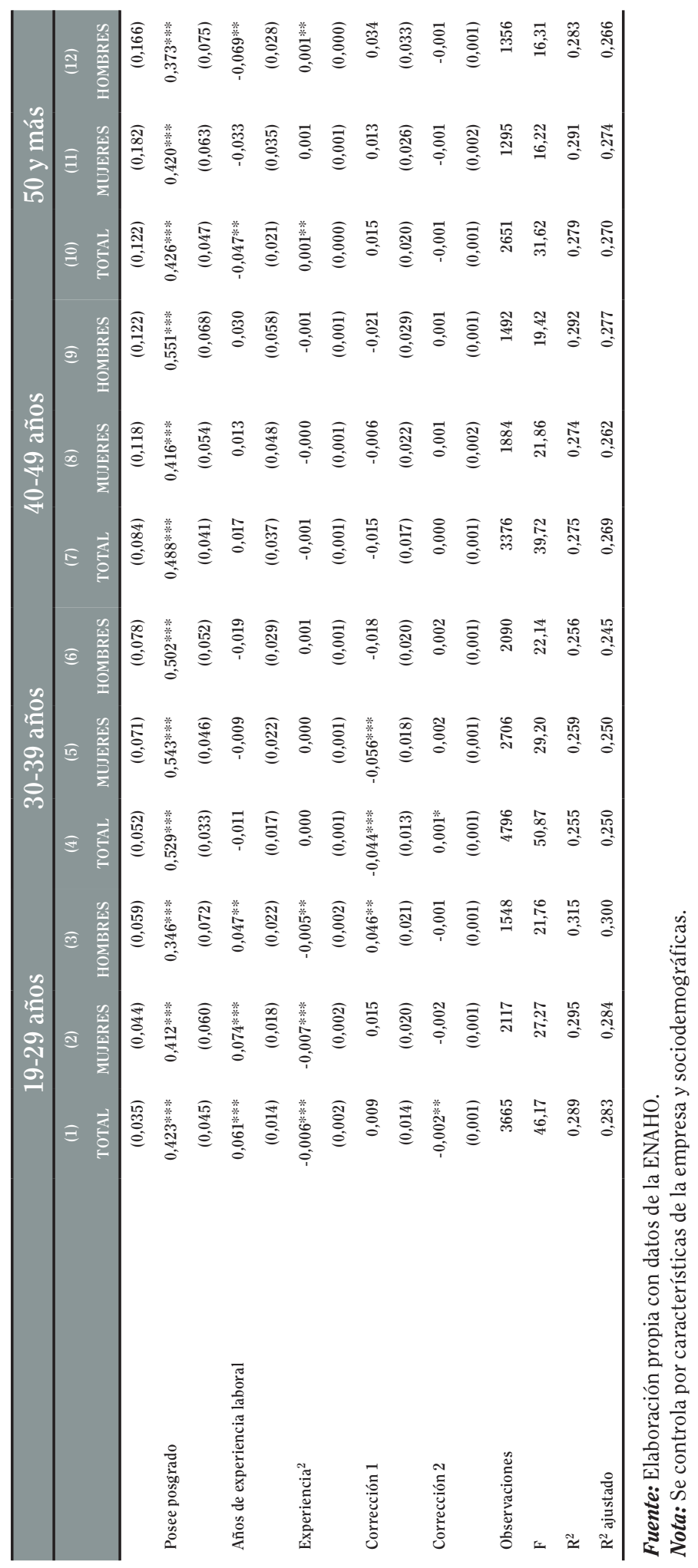




\section{Rendimientos por área de estudio y tipo de universidad}

La tabla 9 muestra los resultados para las regresiones en donde se utilizó una variable categórica para identificar las interacciones entre las áreas de estudio y el tipo de institución. Para este análisis, se realizaron pruebas $t$ para verificar que los salarios por área y tipo de institución fueran estadísticamente diferentes entre sí. $\mathrm{El} \mathrm{R}^{2}$ ronda el $34 \%$ en todos los casos y los modelos son estadísticamente significativos.

Para los hombres en el área de educación, titularse en una universidad privada se asocia con un salario 8,2\% mayor que el de quienes se graduaron de la educación pública, a una significancia del $5 \%$. Para las mujeres, graduarse de una institución privada más bien se asocia con una disminución del 4,5\% en el salario respecto a las mujeres graduadas de educación pública. El efecto positivo del instituto privado, contrario a la hipótesis de la mayor calidad de la educación pública, se puede deber a que el Ministerio de Educación Pública, el mayor contratista de educadores en el país, paga por titulación únicamente. Es decir, todas las personas que tengan un título pueden trabajar en el área y sus salarios están sujetos a los ajustes automáticos del sector público.

En el área de las humanidades, el efecto negativo asociado con dicha área es menor para las instituciones públicas $(-8,6 \%)$ que para las privadas $(-21,2 \%)$ en el modelo agregado. Quienes estudiaron en una universidad pública no reportan, en promedio, un salario diferente a quienes estudiaron educación. Para las personas graduadas de una universidad privada, sin embargo, las mujeres $y$ los hombres en humanidades ganan 8,8\% y 27,4\% menos que sus pares en la categoría de referencia. Es decir, las medias de los salarios tanto para hombres como para mujeres son estadísticamente diferentes entre instituciones privadas y públicas, por lo que se puede afirmar que la penalización por haber estudiado en una universidad privada existe.

La diferencia sobre el salario de los hombres respecto a la categoría de referencia por haber estudiado alguna disciplina social en una universidad pública y una privada es de $8,5 \%$ y $7,1 \%$, respectivamente, al nivel de confianza estándar. Para las mujeres y el modelo agregado, no se observan diferencias con respecto al salario de una persona que haya estudiado educación en una universidad pública.

Con excepción de las mujeres que estudiaron en universidades privadas, el premio por estudiar ciencias básicas y matemáticas es muy alto. Esto puede deberse a que la oferta de dichas carreras es limitada y la mayoría de ellas solo se imparten en las universidades estatales. Para los hombres, el premio por haber estudiado estas carreras en una universidad pública es casi dos veces que el asociado a la institución privada, a un nivel de significancia del 1\%. Para las mujeres, estudiar una de estas carreras en una universidad estatal les puede generar un ingreso $10 \%$ superior al que obtendrían si estudiaran en una universidad privada.

En las ingenierías, arquitectura y manufacturas únicamente se observa un salario promedio superior al de educación si se ha estudiado en una universidad pública. La diferencia salarial respecto a su respectiva categoría base es de 23,1\% para las mujeres y 20,8\% para los hombres, a un nivel de confianza del 99\%. Además, la media de los salarios de las mujeres graduadas de esta área en universidades públicas es estadísticamente diferente (y mayor) a la de las mujeres graduadas de universidades privadas. Igualmente, el salario promedio de quienes estudiaron carreras en el área de agricultura, pesca y ciencias veterinarias es superior al de referencia únicamente para quienes se graduaron de universidades públicas: para las mujeres, esto se asocia con un premio de $27,8 \%$; mientras que, para los hombres, asciende a 13,8\%. A su vez, su media es estadísticamente diferente, $y$ mayor, que la de los hombres graduados de una universidad privada. 
TABLA 9

ECUACIÓN PARA EL LOGARITMO NATURAL DEL SALARIO REAL POR HORA DE LA POBLACIÓN CON ESTUDIOS SUPERIORES, POR GÉNERO (errores estándar robustos) Período: 2011-2017

\begin{tabular}{|c|c|c|c|}
\hline & Total & Mujeres & Hombres \\
\hline \multicolumn{4}{|c|}{ Área de estudio (base = educación pública) } \\
\hline \multicolumn{4}{|l|}{ Educación } \\
\hline \multirow[t]{2}{*}{ Privada } & $-0,016$ & $-0,046^{* *}$ & $0,079 * *$ \\
\hline & $(0,018)$ & $(0,020)$ & $(0,037)$ \\
\hline \multicolumn{4}{|l|}{ Humanidades } \\
\hline \multirow[t]{2}{*}{ Pública } & $-0,090 * *$ & $-0,070$ & $-0,058$ \\
\hline & $(0,042)$ & $(0,056)$ & $(0,067)$ \\
\hline \multirow[t]{2}{*}{ Privada } & $-0,212 * * *$ & $-0,088^{*}$ & $-0,274^{* * * *}$ \\
\hline & $(0,039)$ & $(0,052)$ & $(0,060)$ \\
\hline \multicolumn{4}{|c|}{ Disciplinas sociales } \\
\hline \multirow[t]{2}{*}{ Pública } & 0,023 & $-0,004$ & $0,082^{* * *}$ \\
\hline & $(0,019)$ & $(0,024)$ & $(0,034)$ \\
\hline \multirow[t]{2}{*}{ Privada } & 0,013 & $-0,015$ & $0,069 * *$ \\
\hline & $(0,017)$ & $(0,020)$ & $(0,032)$ \\
\hline \multicolumn{4}{|c|}{ Ciencias, matemática y computación } \\
\hline \multirow[t]{2}{*}{ Pública } & $0,147 * * *$ & $0,099 * *$ & $0,205^{* * *}$ \\
\hline & $(0,026)$ & $(0,040)$ & $(0,040)$ \\
\hline \multirow[t]{2}{*}{ Privada } & $0,060 * *$ & 0,007 & $0,111^{* * *}$ \\
\hline & $(0,026)$ & $(0,044)$ & $(0,040)$ \\
\hline \multicolumn{4}{|c|}{ Ingenierías, arquitectura y manufacturas } \\
\hline \multirow[t]{2}{*}{ Pública } & $0,172^{* * * *}$ & $0,208^{* * * *}$ & $0,189 * * *$ \\
\hline & $(0,028)$ & $(0,053)$ & $(0,041)$ \\
\hline \multirow[t]{2}{*}{ Privada } & 0,043 & 0,025 & $0,070^{*}$ \\
\hline & $(0,028)$ & $(0,047)$ & $(0,041)$ \\
\hline \multicolumn{4}{|c|}{ Agricultura, pesca y ciencias veterinarias } \\
\hline \multirow[t]{2}{*}{ Pública } & $0,104^{* * * *}$ & $0,245^{* * * *}$ & $0,129 * * *$ \\
\hline & $(0,037)$ & $(0,079)$ & $(0,049)$ \\
\hline \multirow[t]{2}{*}{ Privada } & 0,097 & 0,164 & 0,122 \\
\hline & $(0,061)$ & $(0,117)$ & $(0,077)$ \\
\hline \multicolumn{4}{|c|}{ Medicina y estudios relacionados } \\
\hline \multirow[t]{2}{*}{ Pública } & $0,146^{* * * *}$ & $0,103^{* * * *}$ & $0,266^{* * * *}$ \\
\hline & $(0,030)$ & $(0,037)$ & $(0,052)$ \\
\hline \multirow[t]{2}{*}{ Privada } & $-0,062 * * *$ & $-0,116^{* * * *}$ & $0,095^{* * *}$ \\
\hline & $(0,024)$ & $(0,028)$ & $(0,045)$ \\
\hline
\end{tabular}




\begin{tabular}{|c|c|c|c|}
\hline & Total & Mujeres & Hombres \\
\hline \multicolumn{4}{|l|}{ Servicios } \\
\hline \multirow[t]{2}{*}{ Pública } & $-0,089 *$ & $-0,154^{* *}$ & $-0,008$ \\
\hline & $(0,053)$ & $(0,071)$ & $(0,079)$ \\
\hline \multirow[t]{2}{*}{ Privada } & $-0,151^{* * *}$ & $-0,150 * * *$ & $-0,129 * *$ \\
\hline & $(0,041)$ & $(0,054)$ & $(0,064)$ \\
\hline \multicolumn{4}{|c|}{ Calificación (base = educación emparejada) } \\
\hline \multirow[t]{2}{*}{ Aparente sobreeducación } & $-0,279 * * *$ & $-0,249 * * *$ & $-0,315^{* * * *}$ \\
\hline & $(0,010)$ & $(0,013)$ & $(0,015)$ \\
\hline \multirow[t]{2}{*}{ Genuina sobreeducación } & $-0,499 * * *$ & $-0,438 * * *$ & $-0,560 * * *$ \\
\hline & $(0,028)$ & $(0,035)$ & $(0,044)$ \\
\hline \multirow[t]{2}{*}{ Posee posgrado } & $0,504^{* * *}$ & $0,482^{* * *}$ & $0,492^{* * *}$ \\
\hline & $(0,019)$ & $(0,026)$ & $(0,030)$ \\
\hline \multirow[t]{2}{*}{ Años de experiencia laboral } & $0,018^{* * * *}$ & $0,022 * * *$ & $0,016^{* * * *}$ \\
\hline & $(0,002)$ & $(0,002)$ & $(0,002)$ \\
\hline \multirow[t]{2}{*}{ Experiencia $^{2}$} & $-0,000 * * *$ & $-0,000 * * *$ & $-0,000 * * *$ \\
\hline & $(0,000)$ & $(0,000)$ & $(0,000)$ \\
\hline \multirow[t]{2}{*}{ Corrección 1} & $-0,012$ & $-0,014$ & 0,009 \\
\hline & $(0,008)$ & $(0,010)$ & $(0,012)$ \\
\hline \multirow[t]{2}{*}{ Corrección 2} & $-0,000$ & 0,000 & 0,000 \\
\hline & $(0,000)$ & $(0,001)$ & $(0,001)$ \\
\hline Observaciones & 14489 & 8002 & 6487 \\
\hline $\mathrm{F}$ & 192,42 & 106,82 & 85,80 \\
\hline $\mathrm{R}^{2}$ & 0,342 & 0,343 & 0,342 \\
\hline $\mathrm{R}^{2}$ ajustado & 0,340 & 0,340 & 0,338 \\
\hline
\end{tabular}

Fuente: Elaboración propia con base en ENAHO.

Nota: : Se controla por características de la empresa y sociodemográficas. 
Dados los resultados del IFOM, citados previamente, era imaginable que, en efecto, la educación superior pública se relacione con mayores salarios en medicina. Al igual que las personas graduadas en derecho, quienes estudiaron medicina no pueden ejercer sin haber realizado el internado. Esto genera una barrera de entrada a la profesión, de forma que quienes no puedan realizar el internado obtendrán salarios menores a los de las personas médicas. En el caso de las mujeres, no solo estudiar en la universidad pública se asocia con un incremento salarial de $10,8 \%$, sino que hacerlo en una universidad privada reduce el salario promedio en $11 \%$ respecto a la categoría de referencia, a un nivel de confianza de 99\%. Para los hombres, ambos efectos son positivos y significativos al 1\%; sin embargo, el título en universidad pública aporta 17 puntos porcentuales más al premio observado que el título en una universidad privada.

Estudiar un programa en el área de servicios se relaciona con un menor salario relativo a la categoría de referencia en todos los casos, excepto para los hombres graduados de universidades públicas. En el modelo agregado y masculino; sin embargo, el castigo por estudiar en una universidad privada es superior. Para las mujeres no se observa ninguna diferencia salarial según el tipo de institución: ya sea que se haya graduado de una universidad pública o privada, una mujer graduada en servicios gana, en promedio, un salario 13,9\% inferior al de aquella que haya estudiado educación en una universidad pública.

\section{DISCUSIÓN Y CONCLUSIONES}

En este artículo se identificó la sobreeducación para la población graduada de universidades, medida a través de una desviación de la media de años de escolaridad. Se encuentra que, en general, el país no tiene problemas de genuina sobreeducación, ya que la mayoría de las personas graduadas encuentran trabajos que les satisfacen (97,4\%) y no desean cambiarlos (95,6\%), lo que indica que dichos puestos están acordes con sus habilidades. Sin embargo, las tasas de aparente sobreeducación son elevadas. Si bien esto puede asociarse a la construcción del indicador -que toma como referencia la media de escolaridad observada por ocupación-, los datos llaman la atención en diversos sentidos: en primer lugar, podrían estar señalando una sobre inversión en capital humano, producto de la escasez de oferta educativa para desarrollar competencias técnicas $y$ de profesionales medios. También podría señalizar una inflación de las calificaciones, producto del exceso de universidades con una alta concentración de títulos en ciertas áreas como las disciplinas económicas o educación. Pero lo más preocupante es que podría estar reflejando una dualidad en el sistema educativo: al poderse obtener un mismo título con programas que varían considerablemente en extensión, las universidades con los programas más extensos podrían estar sobreeducando a sus estudiantes para el mercado interno y esto podría entrar en conflicto con los objetivos de internacionalización de sus programas, i.e., programas competitivos internacionalmente podrían resultar excesivamente costosos internamente. Esto supone un problema que afecta, en primera instancia, a las universidades públicas, $y$ las obligará a tener que definir una ruta para poder mantener su competitividad internacional y ajustarse a las demandas del mercado interno. Este reto, además, cobra cada vez más fuerza debido a que las universidades privadas insertan una mayor cantidad de personas al mercado laboral. Una posible opción es plantearse la posibilidad de diversificar sus grados académicos para diferenciar sus títulos e introducir diplomados que puedan ser más comparables con los que ofrecen las universidades privadas.

Se estimó también una serie de ecuaciones de salario para analizar la relación existente entre el tipo de universidad y el emparejamiento laboral observado. Los resultados muestran que haber estudiado en una universidad pública sí genera retornos sobre el salario en comparación con haberlo hecho en una universidad privada. Estudiar en una universidad estatal supone un incremento del salario entre 4,7\% y 9,5\% en comparación con quienes, teniendo las mismas características, 
estudiaron en una universidad privada. Lo anterior podría estar reflejando una mejor calidad de la educación en las universidades públicas. Esto sería consistente con que las universidades públicas reciban cerca de la mitad de la matrícula total, pero únicamente representan 37,8\% de los diplomas otorgados (2019), pues podría estar sugiriendo que sus programas poseen un mayor nivel de dificultad académica. Esto podría repercutir, entonces, sobre el desarrollo de mayores habilidades y, consecuentemente, mejores indicadores laborales. Desafortunadamente, con los datos disponibles, no es posible someter a prueba la hipótesis de si las universidades públicas descreman a la población más hábil, pues no se cuenta con información longitudinal sobre esta variable.

El premio asociado a estudiar en una universidad pública es, en general, superior para las mujeres que para los hombres. Además, se puede apreciar que dicho premio se observa principalmente en las cohortes más jóvenes, lo cual era esperable debido a que, al inicio de la vida laboral, el título es lo más relevante a la hora de encontrar trabajo. Por el contrario, dicho premio no se observa en la cohorte de personas con edades superiores a 50 años, probablemente porque, con el transcurrir del tiempo, las personas adquieren experiencia, construyen una reputación individual y no asociada únicamente a su alma mater, $y$ tienen mayores facilidades para moverse dentro del mercado laboral y conseguir un trabajo acorde con sus habilidades.

Por último, se concluye que efectivamente estudiar en centros de educación superior públicos se asocian con mayores ingresos en las áreas de ciencias, matemática y computación; ingenierías, arquitectura y manufacturas; agricultura, pesca y ciencias veterinarias y medicina $y$ estudios relacionados. Lo anterior podría estar reflejando una mayor calidad en su oferta educativa en comparación con los centros privados, que estaría manifiesta en la brecha observada entre las universidades públicas y privadas en las diferentes clasificaciones internacionales de universidades. Es necesario que tales resultados se tomen en cuenta a la hora de discutir políticas asociadas a las universidades, incluido su financiamiento, ya que, si la alternativa privada no es de la misma calidad que la pública, no debería tratárseles como iguales.

Sería deseable también una política educativa que permita realizar mediciones sobre la calidad de las diferentes universidades, con el fin de dar las señales apropiadas al mercado y disminuir la inflación de calificaciones. En particular, porque la inflación de calificaciones podría ser la razón de que se observen castigos tan elevados a la sobreeducación. Para mantener su ventaja, las universidades públicas deberían, además, preocuparse por coordinar mejor con las empresas la colocación de sus estudiantes en el mercado, porque si no se logra dicha diferenciación, sus estudiantes se ven en la obligación de competir por puestos con quienes han invertido menos en educación. Si las empresas ajustan sus puestos de trabajo hacia la media observada $-y$ dado que las universidades públicas producen una minoría de personas graduadas-, más bien podría pagarse un castigo por sobreeducación y, en el largo plazo, el país podría estar haciendo una apuesta hacia un equilibrio de menor nivel.

Una de las mayores limitaciones para estudiar las diferencias en los resultados educativos de quienes estudian en universidades públicas y privadas es la ausencia de estadísticas de las universidades privadas, así como la carencia de bases de datos longitudinales. Este estudio plantea, además, una serie de posibles líneas de investigación futuras, tales como la relación de los indicadores económicos en el mercado laboral con el tipo de educación secundaria pública y privada, $y$ con los salarios para quienes no asistieron a la educación superior, analizar los efectos de la inflación educativa en el mercado laboral, analizar los mecanismos de coordinación de las universidades con las empresas empleadoras de sus personas graduadas, y los efectos de las habilidades para ingresar a una universidad pública y su posible repercusión sobre los salarios. 


\section{REFERENCIAS}

Abarca, A., Leiva, R., Robalino, J., \& Saborío, M. (2016). Diferencias en la permanencia y el desempeño en la educación superior entre estudiantes provenientes de colegios públicos y privados. (Escuela de Economía UCR Serie de documentos de trabajo 16-03). https://web. archive.org/web/20200210172045/http://economia.fce.ucr.ac.cr/es/publicacion/diferencias-enla-permanencia-y-el-desempeno-en-la-educacion-superior-entre-estudiantes

Alpin, C., Shackleton, J.R. \& Walsh, S. (1998). Over- and undereducation in the UK graduate labour market. Studies in Higher Education, 23 (1), 17-34. https://doi.org/10.1080/03075079812331 380462

Anderson, R. E. (1975). Private/public higher education and the competition for high ability students. The Journal of Human Resources, 10(4), 500-511. https://doi.org/10.2307/144987

Arguedas Ortiz, D. (2015, mayo 19). Otto Guevara: Una propuesta "revolucionaria". Semanario Universidad. https://web.archive.org/web/20200210172632/https://historico. semanariouniversidad.com/pais/otto-guevara-una-propuesta-revolucionaria/

Ávalos, A. (2017, diciembre 12). 188 estudiantes de medicina perdieron examen y no harán práctica en hospitales de CCSS. La Nación. https://web.archive.org/web/20171212210921/ https://www.nacion.com/el-pais/salud/188-estudiantes-de-medicina-perdieron-examen-y-no/ XUNWMEM7N5AN3HTBTZNVF47EYE/story/

Ávalos, A. (2018, diciembre 11). Casi 200 alumnos de medicina reprueban examen para hacer práctica en hospitales de CCSS. La Nación. https://web.archive.org/web/20181212052143/ https://www.nacion.com/el-pais/salud/casi-200-alumnos-de-medicina-reprueban-examenpara/OOBI7JNP35BGZEM3GNXWNRAA5E/story/

Becker, G. (1962). Investment in human capital: a theoretical analysis. Chicago: The University of Chicago Press.

Becker, G.S. (1971). The economics of discrimination. Chicago, IL: The University of Chicago Press.

Becker, G. (1993). Human capital. A theoretical and empirical analysis with special reference to education. Chicago: The University of Chicago Press and National Bureau of Economic Research.

Blanco, L. C. (2019) Emparejamiento entre competencias y empleo en el mercado laboral costarricense, Revista de Ciencias Económicas, 37(2), 7-41. https://doi.org/10.15517/rce. v37i2.38868

Blau, F.D. (1999). Women's economic well-being, 1970-1995: Indicators and trends. Focus, 20(1), 4-10.

Blau, F. D., \& Kahn, L. M. (2007). The gender pay gap: have women gone as far as they can? Academy of Management Perspectives, 21(1), 7-23. https://doi.org/10.5465/ amp.2007.24286161

Boll, C., Leppin, J. S., Rossen, A., \& Wolf, A. (2016). Overeducation-New evidence for 25 European countries. (HWWI Research Paper No 173). http://hdl.handle.net/10419/130613

Bruce, M. G. (1983). Private education and the public interest. The Phi Delta Kappan, 65(3), 221-222.

Cerdas, D. (2017, enero 23). 70\% de aspirantes falló examen de incorporación al Colegio de Abogados. La Nación. https://web.archive.org/web/20171109031957/https://www.nacion.com/ el-pais/educacion/70-de-aspirantes-fallo-examen-de-incorporacion-al-colegio-de-abogados/ OHNR74PJ5VH6NMSG64KEPZM6LQ/story/

Cerdas, D., \& Chichilla, S. (2017, junio 7). CONESUP cierra universidad fundada por Justo Orozco debido a irregularidades. La Nación. https://web.archive.org/web/20180905130430/https:// www.nacion.com/el-pais/educacion/conesup-cierra-universidad-fundada-por-justo-orozcodebido-a-irregularidades/M6ROW3GELZE2JEYVCQZQS5X6OY/story/ 
Cerdas, D. (2019, enero 11). El 90\% de graduados de derecho perdió examen de incorporación al Colegio de Abogados. La Nación. https://web.archive.org/web/20190112020753/https:// www.nacion.com/el-pais/educacion/el-90-de-graduados-de-derecho-perdio-examende/6MCTB5OYBNAOTKECVTFV6YOORU/story/

Chevalier, A. (2003). Measuring over-education. Economica, 70(279), 509-531. https://doi. org/10.1111/1468-0335.t01-1-00296

Chevalier, A. (2011). Subject choice and earnings of UK graduates. Economics of Education Review, 30(6), 1187-1201. https://doi.org/10.1016/j.econedurev.2011.04.007

Chevalier, A. \& Lindley, J. (2009). Overeducation and the skills of UK graduates. Journal of the Royal Statistical Society: Series A (Statistics in Society), 172(2), 307-337. https://doi. org/10.1111/j.1467-985X.2008.00578.x

Comisión Nacional de Préstamos para Educación (2017) Presupuesto ordinario 2018. https://web. archive.org/web/20200210174007/http://www.conape.go.cr/wp-content/uploads/2016/09/ Presupuesto-Ordinario-2018.pdf

Consejo Nacional de Rectores (2018, 19 de setiembre). Reglamento orgánico del Consejo Nacional de Rectores y de la Oficina de Planificación de la Educación Superior. La Gaceta, 165

Consejo Nacional de Rectores (2019). Montos FEES: Relación del FEES con el PIB nominal y tasas de crecimiento del FEES 2010-2018. https://web.archive.org/web/20190124183032/http:// siesue.conare.ac.cr/inf-financiera-y-presupuestaria/montos-fees.html

Cybermetrics Lab (2019). Webometrics ranking of world universities. https://web.archive.org/ web/20200206101304/http://www.webometrics.info/en

Davies, J. B., Zhang, J., \& Zeng, J. (2005). Intergenerational mobility under private vs. public education. Scandinavian Journal of Economics, 107(3), 399-417. https://doi.org/10.1111/ j.1467-9442.2005.00415.x

Dolton, P. \& Silles, M. (2001). Over-education in the graduate labour market: some evidence from alumni data. London: Centre for the economics of education, London School of Economics and Political Science.

Doyle, D.P. (1980). Public policy and private education. The Phi Delta Kappan, 62(1), 16-19.

Elsevier B.V. (2019). Scopus. https://web.archive.org/web/20200101070624/https://www.scopus.com/ home.uri

Fernández, A. \& Del Valle, R. (2011). Estimación de los determinantes de la desigual en los ingresos laborales de Costa Rica para el período 2001-2009. Revista de Ciencias Económicas, 29 (2), 229-245. https://revistas.ucr.ac.cr/index.php/economicas/article/view/7022

Fernández, A. \& Del Valle, R. (2013). Desigualdad educativa en Costa Rica: la brecha entre estudiantes de colegios públicos y privados. Análisis con los resultados de la evaluación internacional PISA. Revista CEPAL, 111, 37-57. https://hdl.handle.net/11362/35932

Fioroni, T. (2010). Child mortality and fertility: Public vs private education. Journal of Population Economics, 23(1), 73-97. https://doi.org/10.1007/s00148-009-0248-5

Flores Curiel, D. (2016). Subsidizing or taxing education? A note on the quality gap and government finances. Estudios Económicos, 31(2), 339-353. https://doi.org/10.24201/ee.v31i2.20

Geiger, R. L. (1988). Public and private sectors in higher education: a comparison of international patterns. Higher Education, 17(6), 699-711. https://doi.org/10.1007/BF00143782

Gindling, T. H., \& Trejos, J. D. (2005). Accounting for changing earnings inequality in Costa Rica, 1980-99. The Journal of Development Studies, 41(5), 898-926. https://doi. org/10.1080/00220380500145321 
Ginsburg, M., Brady, K., Draxler, A., Klees, S. J., Luff, P., Patrinos, H. A., \& Edwards, D. (2012). Public-private partnerships and the global reform of education in less wealthy countries-A moderated discussion. Comparative Education Review, 56(1), 155-175. https://doi. org/10.1086/662684

Goldin, C. (2002). A pollution theory of discrimination: male and female differences in occupations and earnings, NBER Working Paper 8985. https://doi.org/10.3386/w8985

Hanushek, E. A. (2002). Publicly provided education, NBER Working Paper 8799. https://doi. org/10.3386/w8799

Hanushek, E. A., Lavy, V., \& Hitomi, K. (2006). Do Students Care about School Quality? Determinants of Dropout Behavior in Developing Countries, NBER Working Paper 12737. https://doi.org/10.3386/w12737

Hanushek, E. A., Ruhose, J., \& Woessmann, L. (2015). Knowledge Capital and Aggregate Income Differences: Development Accounting for U.S. States, NBER Working Paper 21295. https:// doi.org/10.3386/w21295

Heckman, J. J. (1976). The common structure of statistical models of truncation, sample selection and limited dependent variables and a simple estimator for such models. Annals of Economic and Social Measurement, 5(4), 475-492.

Higher Education Statistics Agency (2019). Our vision and principles. https://web.archive.org/ web/20200210175509/https://www.hesa.ac.uk/about/why-we-do-what-we-do

Hodge, G. A., \& Greve, C. (2007). Public-private partnerships: An international performance review. Public Administration Review, 67(3), 545-558. https://doi.org/10.1111/j.15406210.2007.00736.x

Hoyt, W. H., \& Lee, K. (1998). Educational vouchers, welfare effects, and voting. Journal of Public Economics, 69(2), 211-228. https://doi.org/10.1016/S0047-2727(98)00023-1

Instituto Tecnológico de Costa Rica (1983, 24 de enero). Estatuto orgánico del Instituto Tecnológico de Costa Rica. La Gaceta Oficial, 16.

International Labour Organization. (2012). International Standard Classification of Occupations: ISCO-08. International Labour Office.

Levin, H. M. (1991). The economics of educational choice. Economics of Education Review, 10(2), 137-158. https://doi.org/10.1016/0272-7757(91)90005-A

Levy, D. (1982). Private versus public financing of higher education: U.S. policy in comparative perspective. Higher Education, 11(6), 607-628. https://doi.org/10.1007/BF00139778

Machin, S. \& Puhani, P. (2002). Subject of degree and the gender wage differential. Evidence from the UK and Germany, Institute for the Study of Labor IZA discussion paper 553. Institute for the Study of Labor.

Machin, S. \& Puhani, P. (2005). The contribution of degree subject to the gender wage gap for graduates. Preliminary draft. Presentado en Annual Meeting of the Swiss Society of Economics and Statistics on Resource Economics, Technology and Sustainable Development.

Madrigal, L. M. (2015, mayo 14). Otto Guevara: «Tomemos la plata del FEES y se la trasladamos toda a Conape». El Mundo CR. https://web.archive.org/save/https://www.elmundo.cr/costa-rica/ otto-guevara-tomemos-la-plata-del-fees-y-se-la-trasladamos-toda-a-conape/

Marginson, S. (2007). The public/private divide in higher education: A global revision. Higher Education, 53(3), 307-333. https://doi.org/10.1007/s10734-005-8230-y

Mata, G. y Valverde, C. (2006). Rentabilidad de invertir en educación en Costa Rica. Tesis para optar al grado de licenciatura. San José: Universidad de Costa Rica. 
Mayston, D. \& Yang, J. (2008). A pecking order analysis of graduate overeducation and educational investment in China. The University of York Discussion Papers in Economics 2008/25. https://web.archive.org/web/20170814164123/https://www.york.ac.uk/media/economics/ documents/discussionpapers/2008/0825.pdf

McPherson, M. (1981). Quality and competition in public and private higher education. Change: The Magazine of Higher Learning, 13(3), 18-23. https://doi.org/10.1080/00091383.1981.10569802

Mincer, J. (1974). Schooling, experience and earnings. New York: Columbia University Press.

Ministerio de Educación Pública (2001, 11 de julio). Reglamento General del Consejo Nacional de Enseñanza Superior Universitaria Privada, Decreto N²9631-MEP. La Gaceta, 133.

Montero, E., Villalobos, J. y Valverde, A. (2007). Factores institucionales, pedagógicos, psicosociales y sociodemográficos asociados al rendimiento académico en la Universidad de Costa Rica: Un análisis multinivel. Revista Electrónica de Investigación y Evaluación Educativa, 13(2), 215234.

Montero, E., Rojas, S., Zamora, E. y Rodino, A.M. (2012). Costa Rica en las pruebas PISA 2009 de comprensión lectora y alfabetización matemática. En: PEN (ed.). Cuarto Informe del Estado de la Educación Costarricense. San José: PEN.

Mumford, K., \& Smith, P. N. (2009). What determines the part-time and gender earnings gaps in Britain: Evidence from the workplace. Oxford Economic Papers, 61(suppl_1), i56-i75. https:// doi.org/10.1093/oep/gpn041

Oaxaca, R. (1973). Male-female wage differentials in urban labor markets. International Economic Review, 14(3), 693-709. https://doi.org/10.2307/2525981

Paquette, J. (2005). Public funding for "private" education: the equity challenge of enhanced choice. American Journal of Education, 111(4), 568-595. https://doi.org/10.1086/431184

Patrinos, H.A., Barrera-Osorio, F. y Guáqueta, J. (2009). The role and impact of public-private partnerships in education. The World Bank. https://doi.org/10.1596/978-0-8213-7866-3

Programa Estado de la Nación en Desarrollo Humano Sostenible (2015). Quinto informe del estado de la educación costarricense. San José: PEN.

Programa Estado de la Nación en Desarrollo Humano Sostenible (2019). Sétimo informe del estado de la educación costarricense. San José: PEN.

Quacquarelli Symonds Limited. (2019). QS Latin American university rankings 2018. https://web. archive.org/web/20181115091358/https://www.topuniversities.com/university-rankings/latinamerican-university-rankings/2018

Quintini, G. (2011). Over-qualified or under-skilled: a review of existing literature. OECD Social, Employoment and Migration Working Papers, 121. https://doi.org/10.1787/5kg58j9d7b6d-en

Research Excellence Framework (2019). What is the REF? https://web.archive.org/ web/20190619222818/https://www.ref.ac.uk/about/what-is-the-ref/

Reisz, R. D., \& Stock, M. (2012). Private higher education and economic development. European Journal of Education, 47(2), 198-212. https://doi.org/10.1111/j.1465-3435.2012.01518.x

Rosenfeld, R. A. (1979). Women's occupational careers: individual and structural explanations. Sociology of Work and Occupations, 6(3), 283-311. https://doi. org/10.1177/073088847900600302

Sandy, J., \& Duncan, K. (1996). Does private education increase earnings? Eastern Economic Journal, 22(3), 303-312. JSTOR

Schultz, T. W. (1961). Investment in human capital. The American Economic Review, 51(1), 1-17. JSTOR. 
Sequeira, A. (2018, 3 de diciembre). Reforma fiscal aprobada en forma definitiva en la Asamblea con 34 votos, La Nación. https://web.archive.org/web/20200109141806/https://www. nacion.com/el-pais/politica/reforma-fiscal-aprobada-en-forma-definitiva-en-la/ SETGHL7VHFBBHLXFLGXBJJYQYQ/story/

Sexton, E. A. y Nickel, J.F. (1992). The effects of school location on the earnings of black and white youths. Economics of Education Review, 11(1), 11-18. https://doi.org/10.1016/02727757(92)90017-W

Solís, M. I. (2018, diciembre 18). 34 estudiantes de medicina obtuvieron puntaje mayor a 600 en prueba IFOM. https://web.archive.org/web/20200217165343/https://www.ccss.sa.cr/ noticia?34-estudiantes-de-medicina-obtuvieron-puntaje-mayor-a-600-en-prueba-ifom

Stiglitz, J. E. (1974). The demand for education in public and private school systems. Journal of Public Economics, 3(4), 349-385. https://doi.org/10.1016/0047-2727(74)90005-X

Stotsky, J. (2006). Gender and its relevance to macroeconomic policy: a survey. IMF. Recuperado el 17 de junio de 2019 de

https://www.imf.org/en/Publications/WP/Issues/2016/12/31/Gender-and-its-Relevance-toMacroeconomic-Policy-A-Survey-19890

Universidad Estatal a Distancia (2000, 20 de octubre). Estatuto orgánico de la Universidad de Estatal a Distancia. La Gaceta Oficial, 201.

Universidad de Costa Rica, Consejo Universitario (1974, 22 de marzo). Estatuto orgánico de la Universidad de Costa Rica. La Gaceta Oficial, 56.

Universidad de Costa Rica, Escuela de Psicología (2017). Plan de estudios bachillerato y licenciatura en psicología (modificaciones 2015). Recuperado el 25 de febrero de 2019 de https:// web.archive.org/web/20200217165837/https://www.psico.ucr.ac.cr/images/Documentos/ PlanVigente.pdf

Universidad Latina de Costa Rica. (2019). Licenciatura en psicología. https:/web.archive.org/save/ https://www.ulatina.ac.cr/oferta-academica/ciencias-de-la-salud/psicologia?hsCtaTracking=1a ee65dc-a570-45aa-967e-e536859732e7\%7C76d51e58-04f1-4fe5-83db-e40bfbb81979

Universidad Nacional. (2015, 12 de junio). Estatuto orgánico de la Universidad Nacional. La Gaceta Oficial, 113.

Universidad Técnica Nacional. (2010, 28 de junio). Estatuto orgánico de la Universidad Técnica Nacional. La Gaceta Oficial, 124.

Verdugo, R. R., \& Verdugo, N. T. (1988). Overeducation and the earnings of black, hispanic, and white male workers. Sociological Perspectives, 31(2), 190-212. https://doi. org/10.2307/1389082

Verhaest, D., \& Omey, E. (2006). The impact of overeducation and its measurement. Social Indicators Research, 77(3), 419-448. https://doi.org/10.1007/s11205-005-4276-6

Vizcaíno, I. \& Cerdas, D. (2018, 18 de diciembre). Conesup ordena el cierre definitivo de la Universidad Cristiana del Sur. La Nación. https://web.archive.org/web/20190131201214/ https://www.nacion.com/el-pais/educacion/conesup-ordena-el-cierre-definitivo-de-la/ ZVKUYEZSCREHHCZKCHKN74GS3Q/story/

Welch, A. R. (2007). Blurred vision?: Public and private higher education in Indonesia. Higher Education, 54(5), 665-687. https://doi.org/10.1007/s10734-006-9017-5

White, L. (2003). Deconstructing the public-private dichotomy in higher education. Change: The Magazine of Higher Learning, 35(3), 48-54. https://doi.org/10.1080/00091380309604102 